\title{
Adenovirus vector vaccination reprograms pulmonary fibroblastic niches to support protective inflating memory $\mathrm{CD} 8^{+} \mathrm{T}$ cells
}

\author{
Jovana Cupovic ${ }^{1,2}$, Sandra S. Ring1, Lucas Onder1, Julia M. Colston $\mathbb{1}^{3}$, Mechthild Lütge ${ }^{1}$, \\ Hung-Wei Cheng ${ }^{1}$, Angelina De Martin', Nicholas M. Provine $\mathbb{C}^{3}$, Lukas Flatz ${ }^{1,4}$, Annette Oxenius $\mathbb{D}^{5}$, \\ Elke Scandella', Philippe Krebs $\mathbb{1}^{6}{ }^{6}$, Daniel Engeler ${ }^{7}{ }^{7}$, Paul Klenerman ${ }^{3,9 凶}$ and Burkhard Ludewig ${ }^{1,8,9 凶}$
}

\begin{abstract}
Pathogens and vaccines that produce persisting antigens can generate expanded pools of effector memory CD8 ${ }^{+} \mathbf{T}$ cells, described as memory inflation. While properties of inflating memory $\mathrm{CD}^{+} \mathbf{T}$ cells have been characterized, the specific cell types and tissue factors responsible for their maintenance remain elusive. Here, we show that clinically applied adenovirus vectors preferentially target fibroblastic stromal cells in cultured human tissues. Moreover, we used cell-type-specific antigen targeting to define critical cells and molecules that sustain long-term antigen presentation and $\mathrm{T}$ cell activity after adenovirus vector immunization in mice. While antigen targeting to myeloid cells was insufficient to activate antigen-specific CD8 ${ }^{+} \mathbf{T}$ cells, genetic activation of antigen expression in Ccl19-cre-expressing fibroblastic stromal cells induced inflating $\mathrm{CDB}^{+} \mathrm{T}$ cells. Local ablation of vector-targeted cells revealed that lung fibroblasts support the protective function and metabolic fitness of inflating memory $\mathrm{CDB}^{+} \mathrm{T}$ cells in an interleukin (IL)-33-dependent manner. Collectively, these data define a critical fibroblastic niche that underpins robust protective immunity operating in a clinically important vaccine platform.
\end{abstract}

$\mathrm{T}$ he ultimate goal of T cell-based vaccination strategies is the induction of long-term immunological protection via effector memory T cells ${ }^{1}$. Adenovirus (Ad) vector-based vaccines using the backbone of human Ads have been shown in clinical studies to be highly immunogenic with induction of specific antibody and/or $\mathrm{T}$ cell responses to viral ${ }^{2,3}$ or cancer antigens ${ }^{4}$. However, pre-existing anti-Ad antibodies in vaccinated individuals reduces immunogenicity and efficacy of vaccines based on human $\mathrm{Ads}^{2,4}$, which led to the development of non-human vectors based on chimpanzee viruses ${ }^{5}$. Chimeric Ad vectors have been shown to induce long-lived $\mathrm{T}$ cell responses against severe acute respiratory syndrome coronavirus 2 (SARS-CoV-2) ${ }^{6,7}$ and to generate polyfunctional central and effector memory $\mathrm{T}$ cells against hepatitis $\mathrm{C}$ virus $^{8}$. Other approaches to circumvent pre-existing anti-Ad vector immunity include the use of viral backbones with rare serotypes such as Ad26 (ref. ${ }^{3}$ ) or prime-boost regimen with recombinant (r) Ad26-S given on day 0 and rAd5-S given on day 21 ( ref. $^{9}$ ). Although the clinical development of Ad-based vaccines is well advanced, the basic features that determine the high immunogenicity of these viral vectors are still largely unclear.

One of the prominent qualities of Ad vectors that can guide their further improvement is the generation of inflating memory $\mathrm{CD} 8^{+}$ $\mathrm{T}$ cells ${ }^{10}$. Steadily growing memory CD8 ${ }^{+} \mathrm{T}$ cell pools were initially described for the murine cytomegalovirus (MCMV) infection ${ }^{11-13}$, a feature that is most likely associated with the continued generation of antigenic peptides during viral reactivation in peripheral tissues $^{14}$. The persistent expansion of effector memory $\mathrm{T}$ cell populations (that is, 'high' for receptors CX3CR1 and KLRG1 and 'low' for chemokine receptor CCR7 and markers CD62L, CD28, CD27, CD127) occurs not only during MCMV infection ${ }^{15}$ but is one of the hallmarks of an inflationary $\mathrm{CD}^{+} \mathrm{T}$ cell response against human cytomegalovirus $(\mathrm{CMV})^{16,17}$. Use of replication-defective, rAd5-based vectors has further guided the molecular characterization of inflationary $\mathrm{CD}^{+} \mathrm{T}$ cell memory. Immunization with $\beta$-galactosidase (bgal)-expressing Ad results in long-lasting transduction of cells in peripheral organs such as the liver and lungs, leading to the generation of antigenic peptides for several months ${ }^{18}$. The transgene encoding bgal harbors sequences for two $\mathrm{H} 2-\mathrm{K}^{\mathrm{b}}$-restricted antigenic peptides, one epitope generating an expanding-contracting central memory $\mathrm{CD} 8^{+} \mathrm{T}$ cell pool and the second epitope producing an inflating effector memory $\mathrm{T}$ cell response ${ }^{18,19}$. The continuously expanding $\mathrm{CD}^{+} \mathrm{T}$ cell population depends on the presence of major histocompatibility complex (MHC) II-positive cells, while antigen processing in professional antigen-presenting cells seems to be dispensable ${ }^{18}$. Thus, it appears that antigen presentation by non-hematopoietic cells is crucial for the generation of inflating memory $\mathrm{CD}^{+} \mathrm{T}$ cell responses following Ad vector immunization ${ }^{18,20}$. However, the nature of such long-lived, non-hematopoietic cells has remained elusive.

Lymphoid organ stromal cells, that is, lymphatic and blood endothelial cells and fibroblastic reticular cells (FRCs), generate not only the infrastructure for lymphocyte entry and exit but also provide dedicated niches for lymphocyte activation, differentiation and maintenance ${ }^{21-23}$. During MCMV infection, it is assumed that antigen-presenting stromal cells drive memory inflation through interaction with cross-presenting dendritic cells (DCs) in lymphoid

'Institute of Immunobiology, Kantonsspital St. Gallen, St. Gallen, Switzerland. ${ }^{2}$ Max Planck Institute for Immunobiology and Epigenetics, Freiburg, Germany. ${ }^{3}$ Peter Medawar Building for Pathogen Research, Nuffield Department of Medicine, University of Oxford, Oxford, UK. ${ }^{4}$ Department of Dermatology, University Hospital Zurich, Zurich, Switzerland. Institute of Microbiology, ETH Zurich, Zurich, Switzerland. ${ }^{\circ}$ Institute of Pathology, University of Berne, Berne, Switzerland. ${ }^{7}$ Department of Urology, Kantonsspital St. Gallen, St. Gallen, Switzerland. ${ }^{8}$ Institute of Experimental Immunology, University of Zurich, Zurich, Switzerland. ${ }^{9}$ These authors contributed equally: Paul Klenerman, Burkhard Ludewig. ${ }^{凶}$ e-mail: paul.klenerman@medawar.ox.ac.uk; burkhard.ludewig@kssg.ch 

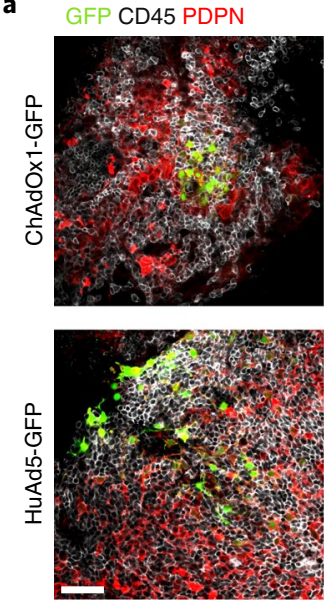
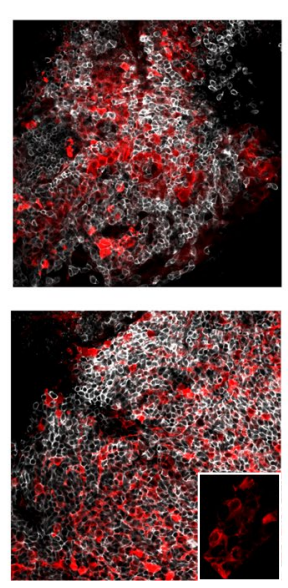
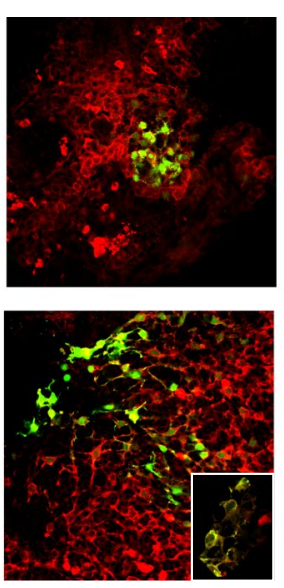
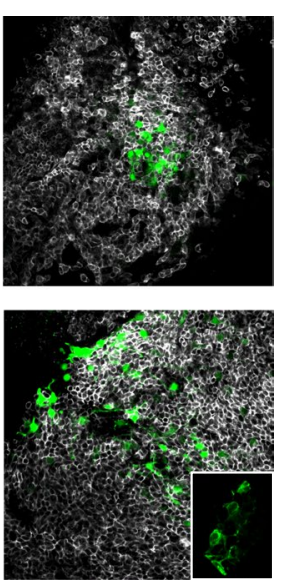

b

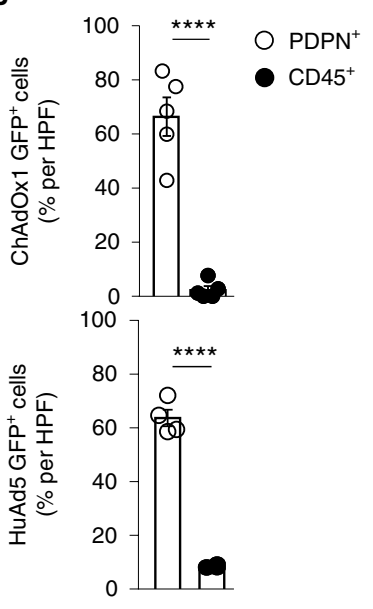

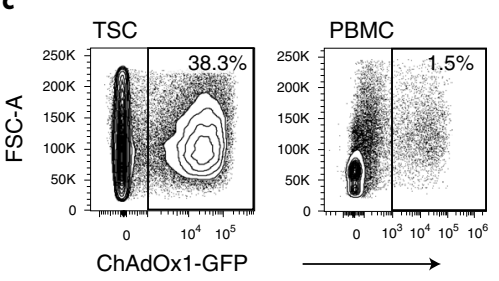

d

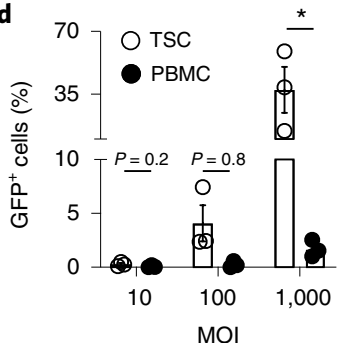

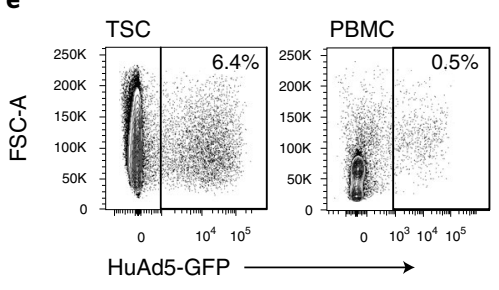

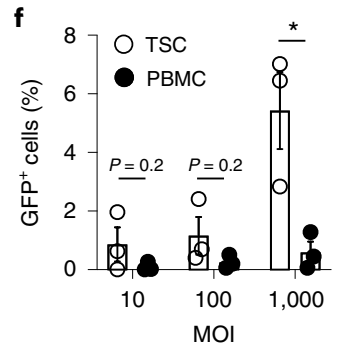

Fig. 1 Ad vectors target human fibroblasts. a,b, Infection of sliced tissue cultures from human palatine tonsils with ChAdOx1-GFP or HuAd5-GFP. Representative high-resolution immunofluorescence images (a) and frequency of GFP+ cells within $\mathrm{CD}^{+} 5^{+}$cells or PDPN ${ }^{+}$cells per high-power field (HPF) (b). Scale bar, $50 \mu \mathrm{m}$. c-f, Infection of cultured TSCs or PBMCs with ChAdOx1-GFP (c,d) or HuAd5-GFP (e,f) at different MOI. d,f, Frequency of GFP+ cells. Dots represent individual samples, and lines indicate mean \pm s.e.m. Pooled data from four independent experiments with $n=5$ ChAdOx $1-G F P$ and $n=4$ HuAd5-GFP tonsillar adenoid tonsil samples (b) and three independent experiments with $n=3$ TSC samples or two independent experiments with $n=3$ PBMC samples (d-f). Statistical analysis was performed using unpaired two-tailed Student's $t$-test $(\mathbf{b}, \mathbf{d}, \mathbf{f})$ with ${ }^{\star} P<0.05,{ }^{\star \star \star \star} P<0.0001$. Exact $P$ values are provided in the source data.

organs $^{24-26}$. In addition, stromal cells can provide key growth factors, such as IL-15, for the long-term sustenance of inflating memory $\mathrm{T}$ cells ${ }^{27}$. Here, we describe that clinically relevant Ad vaccine vectors preferentially target fibroblastic stromal cells (FSCs) in explant cultures of human tonsillar tissue. The target cell selectivity of human (Hu)Ad5-based vectors for podoplanin (PDPN)-expressing FSCs was confirmed in murine models that restrict Ad vector-mediated transgene expression to Cre recombinase-positive cells. We found that long-term transduction of Ccl19-cre ${ }^{+}$FSCs in the lung was crucial for the induction and sustenance of inflating memory $\mathrm{CD}^{+}$ $\mathrm{T}$ cells, which can protect the host from metastasizing cancer. Importantly, adenovirus-based vaccination induced reprogramming of pulmonary FSCs that fostered the generation of a distinct IL-33-producing FSC subset. In sum, adenoviral vectors transform the pulmonary fibroblastic landscape and thereby generate microenvironmental niches that are crucial for the maintenance of protective inflationary memory $\mathrm{CD}^{+} \mathrm{T}$ cells.

\section{Results}

Targeting of human fibroblasts by Ad vectors. Human Ads were first described as cytopathogenic agents isolated from human adenoid tonsils ${ }^{28}$. Here, we used sliced tissue cultures from human palatine tonsils to assess the cellular tropism of green fluorescent protein (GFP)-expressing Ad vectors (Extended Data Fig. 1a). Both HuAd5-GFP and chimpanzee (Ch)AdOx1-GFP vectors readily transduced cells in the tissue slides, with $\mathrm{PDPN}^{+} \mathrm{CD} 45^{-}$cells most frequently expressing the GFP transgene (Fig. 1a,b). Next, we compared side-by-side the susceptibility to infection with recombinant
Ad vectors of short-term cultured tonsillar stromal cells (TSCs) and peripheral blood mononuclear cells (PBMCs). Both ChAdOx1-GFP (Fig. 1c,d) and HuAd5-GFP (Fig. 1e,f) efficiently transduced TSCs following an incubation of $3 \mathrm{~h}$, while the transduction rate of PBMCs remained low. Even at the high multiplicity of infection (MOI) of 1,000 particles per cells, less than $2 \%$ of the hematopoietic cells were GFP-positive (Fig. 1d,f). Those hematopoietic cells that expressed the viral transgene were mainly CD14 ${ }^{+} \mathrm{HLA}-\mathrm{DR}^{+}$monocytes (Extended Data Fig. 1b-d). The majority of $\mathrm{GFP}^{+}$cells in cultured TSCs were $\mathrm{PDPN}^{+}$fibroblasts with only few $\mathrm{CD} 31^{+}$endothelial cells harboring the transgene following exposure to ChAdOx1-GFP (Extended Data Fig. 1e,f) or HuAd5-GFP (Extended Data Fig. 1e,g). In addition, both ChAdOx1 and HuAd5 vectors delivered the transgene to $\mathrm{PDPN}^{+}$fibroblasts in cultured human skin stromal cell (Extended Data Fig. 1h-j) and cultured lung stromal cell preparations (Extended Data Fig. 1k,m). These data demonstrate that clinically relevant Ad vectors efficiently transduce human fibroblasts derived from both lymphoid and non-lymphoid organs.

FSC-dependent induction of inflating memory $\mathrm{CD8}^{+} \mathrm{T}$ cells. HuAd5-based vectors expressing the bgal antigen elicit inflating memory $\mathrm{CD}^{+} \mathrm{T}$ cell responses in mice with up to $30 \%$ of $\mathrm{CD}^{+}$ $\mathrm{T}$ cells in peripheral blood and up to $40 \%$ of $\mathrm{CD}^{+} \mathrm{T}$ cells in lungs recognizing the bgal $_{96}$ epitope, while the bgal $_{497}$ epitope generates a classical contracted memory $\mathrm{CD}^{+} \mathrm{T}$ cell population (Extended Data Fig. 2a-d and ref. ${ }^{18}$ ). To restrict transgene expression to specific cell types in mice, we engineered a cassette with double-floxed Cre recombinase recognition sites in inverted orientation flanking the 
a Ad-LacZ/FlexON

b

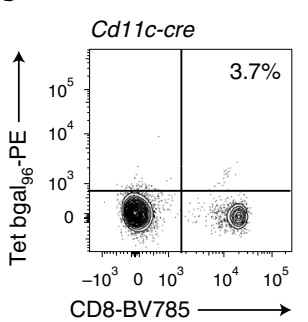

f

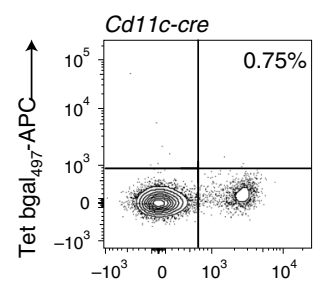

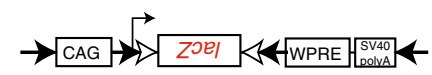

c
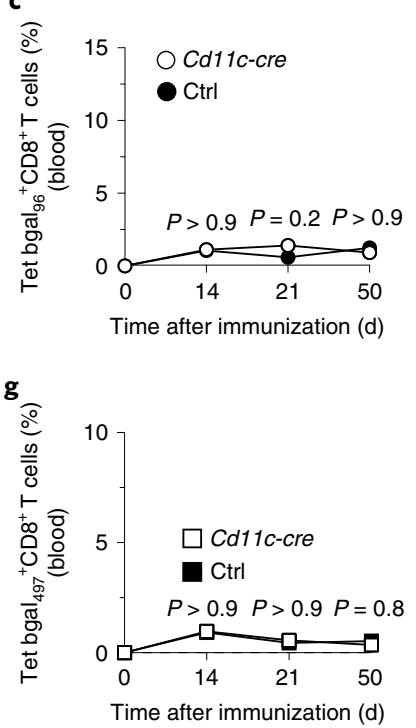

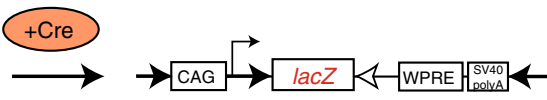

d
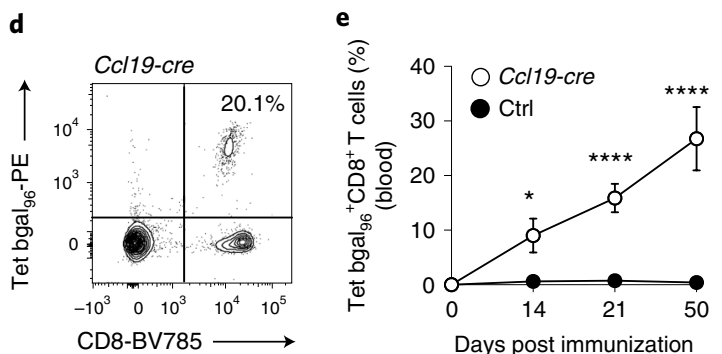

h

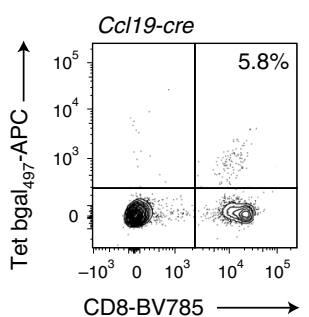

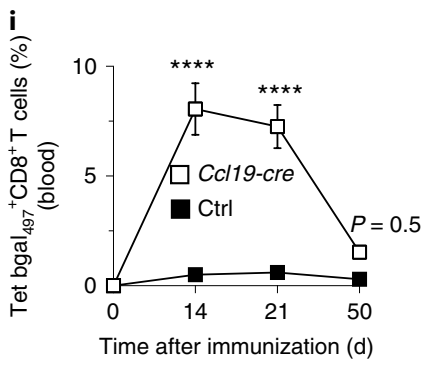

Fig. 2 | Ccl19-cre ${ }^{+}$FSCs mediate induction of inflationary memory CD8+ $\mathbf{T}$ cells. a, Schematic depiction of HuAd5-based vectors containing a cassette with double-floxed Cre recombinase recognition sites with inverted orientation flanking the lacZ gene. Cell-type-specific expression of Cre recombinase induces bgal antigen expression (Ad-LacZ/FlexON). WPRE, woodchuck hepatitis virus post-transcriptional regulatory element. b-i, Kinetics of the frequency of bgal $_{96}$ tetramer ${ }^{+} \mathrm{CD} 8^{+} \mathrm{T}$ cells $(\mathbf{b}-\mathbf{e})$ and bgal ${ }_{497}$ tetramer ${ }^{+} \mathrm{CD} 8^{+} \mathrm{T}$ cells $(\mathbf{f}-\mathbf{i})$ with representative flow cytometry plots in $\mathrm{Cd}$ (11c-cre $(\mathbf{b}, \mathbf{f})$ and Ccl19-cre mice (d,h). Tet, tetramer. Cre-negative mice were used as controls (Ctrl). Values indicate mean \pm s.e.m. for each time point. Pooled data from two independent experiments with $n=8$ (Ctrl) and $n=11$ (Cd11c-cre) mice (c,g); or $n=12$ (Ctrl) and $n=12$ (Ccl19-cre) mice (e,i). Statistical analysis was performed using two-way ANOVA with Bonferroni's multiple-comparisons test $(\mathbf{c}, \mathbf{g}, \mathbf{e}, \mathbf{i})$ with ${ }^{\star} P<0.05,{ }^{\star \star} P<0.01,{ }^{\star \star \star} P<0.001,{ }^{\star \star \star \star} P<0.0001$. Exact $P$ values are provided in the source data.

lacZ gene ${ }^{29}$ and generated a HuAd5-based vector termed Ad-LacZ/ FlexON (Fig. 2a). Injection of Ad-LacZ/FlexON into mice with ubiquitous Cre recombinase expression led to the inflation of bgal ${ }_{96}$ epitope-specific $\mathrm{CD}^{+} \mathrm{T}$ cells (Extended Data Fig. 2e). To probe the ability of different myeloid cell populations to induce and expand inflating memory $\mathrm{CD}^{+} \mathrm{T}$ cell pools in response to Ad vector vaccination, we immunized Cd11c-cre and LysM-cre mice with Ad-LacZ/ FlexON. We found that the frequency of bgal ${ }_{96}$-specific $\mathrm{CD}^{+} \mathrm{T}$ cells in mice with Cre recombinase expression in CD11 $c^{+}$DCs (Fig. 2b,c) or $\mathrm{LysM}^{+}$macrophages or neutrophils (Extended Data Fig. 2f,g) was not substantially elevated compared with that in Cre-negative control mice. In stark contrast, mice with Cre recombinase expression in Ccl19-cre ${ }^{+}$FRCs in lymphoid organs ${ }^{30,31}$ and dedicated niches in peripheral organs $s^{32,33}$ showed pronounced expansion of bgal $_{96}$ epitope-specific CD8 ${ }^{+} \mathrm{T}$ cells (Fig. $2 \mathrm{~d}$,e). Neither Cd11c-cre nor LysM-cre mice showed substantial activation of bgal $_{497}$-specific $\mathrm{CD}^{+} \mathrm{T}$ cells (Fig. 2f,g and Extended Data Fig. 2g), whereas expanding-contracting $\mathrm{CD}^{+} \mathrm{T}$ cell pools reactive to the bgal $_{497}$ epitope were induced in Ccl19-cre mice (Fig. 2h,i). Collectively, our findings reveal that Ad vectors efficiently target FSCs and that particular FSC populations, highlighted by the Ccl19-cre transgene in mice, are crucial for the induction of memory $\mathrm{CD}^{+} \mathrm{T}$ cell inflation.

FSC-DC cooperation supports inflating memory $\mathrm{CD8}^{+} \mathrm{T}$ cells. Next, we investigated whether $\mathrm{Ccl} 19-\mathrm{cre}^{+}$cells are sufficient to induce inflating memory $\mathrm{CD}^{+} \mathrm{T}$ cells after Ad vector immunization. In the first set of experiments, we restricted the peptidepresentation capacity to non-hematopoietic cells through the transfer of $\mathrm{H} 2-\mathrm{K}^{\mathrm{b}}$-deficient bone marrow into sublethally irradiated Ccl19-cre mice (Fig. 3a). We found that control mice with MHC
I-competent bone marrow cells generated inflating bgal ${ }_{96}$-specific $\mathrm{CD}^{+}$memory $\mathrm{T}$ cells (Fig. $3 \mathrm{~b}, \mathrm{c}$ ) in magnitude and kinetics that were comparable with those of non-irradiated Ccl19-cre mice (Fig. 2e and Extended Data Fig. 2a-c). By contrast, memory inflation of bgal ${ }_{96}$-specific CD8 ${ }^{+} \mathrm{T}$ cells was almost completely blocked in Ccl19-cre mice harboring $\mathrm{H} 2-\mathrm{K}^{\mathrm{b}}$-deficient hematopoietic cells (Fig. 3b,c). Likewise, bone marrow chimeric Ccl19-cre mice with a deficiency in cross-presenting DCs, due to the lack of basic leucine zipper ATF-like transcription factor 3 (BATF3) ${ }^{34}$ in their hematopoietic compartment (Fig. 3d), failed to generate inflating bgal ${ }_{96}$-specific $\mathrm{CD}^{+}$memory $\mathrm{T}$ cells (Fig. $3 \mathrm{e}, \mathrm{f}$ ). The impaired cross-presentation due to the absence of $\mathrm{H}_{2}-\mathrm{K}^{b}$ (Extended Data Fig. 3a) or Batf3 expression (Extended Data Fig. 3b) in the hematopoietic compartment of bone marrow chimeric Ccl19-cre mice almost completely abolished the expansion of bgal ${ }_{497}$-specific CD8 ${ }^{+}$ $\mathrm{T}$ cells. These results indicate that $\mathrm{Ccl19-}-\mathrm{cre}^{+} \mathrm{FSCs}$ require cooperation with MHC class I-competent, cross-presenting DCs to facilitate priming and expansion of inflationary memory $\mathrm{CD}^{+} \mathrm{T}$ cells after immunization with bgal-recombinant Ad vectors.

Pulmonary Ccl19-cre ${ }^{+}$FSCs support inflationary $\mathrm{CD8}^{+} \mathrm{T}$ cells. Following intravenous application, the Ad-LacZ vector transduces target cells mainly in the liver, lungs and spleen ${ }^{18,35}$. As Ad-LacZ/ FlexON vaccination of splenectomized Ccl19-cre mice did not significantly alter the inflationary $\mathrm{CD}^{+}$cell response to the bgal ${ }_{96}$ epitope (Extended Data Fig. 4a), we concluded that FSCs mediating memory inflation most likely reside in non-lymphoid organs.

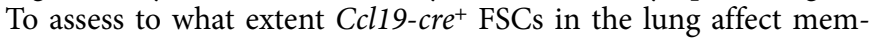
ory inflation, we used an organ-specific cell ablation protocol in Ccl19 $9^{\mathrm{EYFP} / \mathrm{iDTR}} \mathrm{mice}^{33}$, which highlights Ccl19-cre lineage-positive 
a

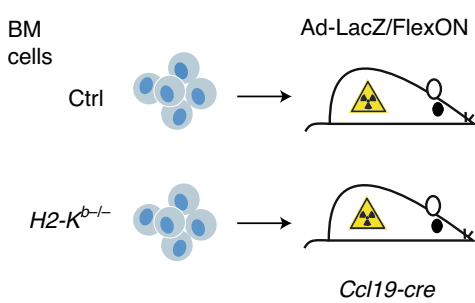

d

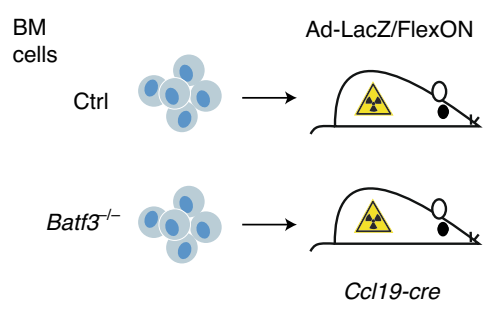

b

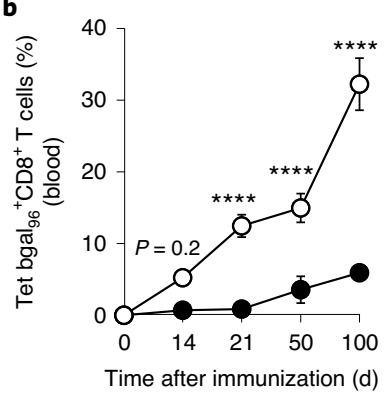

e

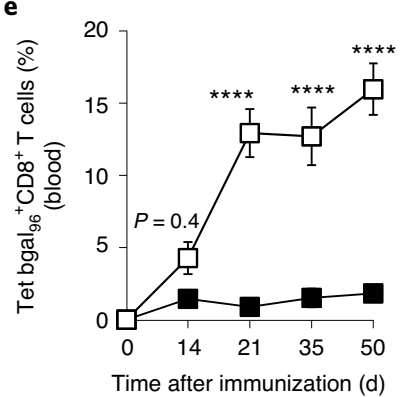

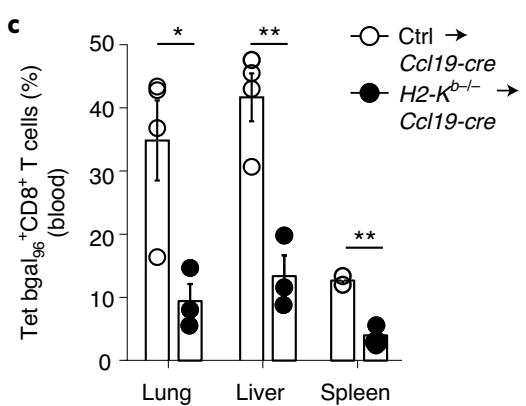

f

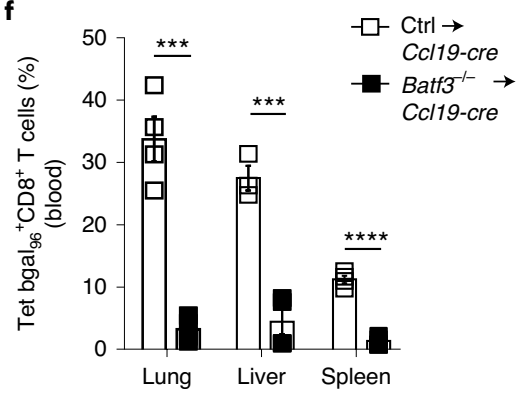

Fig. 3 | Ccl19-cre ${ }^{+}$FSCs collaborate with BATF3 ${ }^{+}$cross-presenting DCs for the induction of LacZ-specific CD8 ${ }^{+} \mathrm{T}_{\text {cell }}$ responses. Bone marrow chimeric mice were generated by transferring $\mathrm{H} 2-\mathrm{K}^{\mathrm{b}}$-deficient (a-c) or BATF3-deficient ( $\mathbf{d}-\mathbf{f}$ ) bone marrow into sublethally irradiated $\mathrm{Ccl19}$-cre mice that were vaccinated intravenously (i.v.) with Ad-LacZ/FlexON. b,e The frequency of bgal ${ }_{96}$ tetramer ${ }^{+} \mathrm{CD} 8^{+} \mathrm{T}$ cells was monitored in blood. $\mathbf{c , f}, \mathrm{f}_{1}$ On day 50 , bgal ${ }_{96}$ tetramer ${ }^{+} \mathrm{CD} 8{ }^{+} \mathrm{T}$ cells in the lung, liver and spleen were analyzed. Pooled data from two independent experiments with $n=10(\mathrm{Ctrl}$ to $\mathrm{Ccl} 19-\mathrm{cre})$ to $n=8$

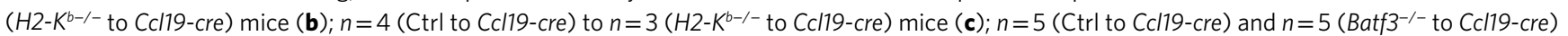
mice (e); and $n=4$ (Ctrl to Ccl19-cre) and $n=4$ (Batf3-/- to Ccl19-cre) mice (f). Values indicate mean \pm s.e.m. for each time point or organ analyzed. Statistical analysis was performed using two-way ANOVA with Bonferroni's multiple-comparisons test (b,e) or unpaired two-tailed Student's $t$-test (c,f) with ${ }^{\star} P<0.05,{ }^{\star \star} P<0.01,{ }^{\star \star \star} P<0.001,{ }^{\star \star \star \star} P<0.0001$. Exact $P$ values are provided in the source data.

cells with the fluorescent marker EYFP and facilitates ablation of genetically tagged cells by intranasal (i.n.) application of diphtheria toxin (DT) (Extended Data Fig. 4b,c). Ccl19-cre ${ }^{+}$FSCs in lung tissue include smooth muscle actin ACTA2 ${ }^{+}$vascular smooth muscle or adventitial cells (Fig. 4a). DT application (i.n.) significantly reduced the number of EYFP ${ }^{+}$cells in the lung (Fig. $4 \mathrm{a}$ and Extended Data Fig. 4c), whereas transgene expression in other organs such as the mediastinal lymph node (Fig. 4a), liver and spleen (Extended Data Fig. 4d) was not affected. By contrast, systemic DT application via intraperitoneal injection led to almost complete ablation of Ccl19-cre ${ }^{+}$FSCs cells in the lung, mediastinal lymph node (Fig. 4a), liver and spleen (Extended Data Fig. 4d). As described previously ${ }^{36}$, ablation of Ccl19-cre ${ }^{+}$cells in lymph nodes following systemic DT application precipitated impaired immune cell organization and cellularity (Fig. 4a). Consequently, systemic ablation of Ccl19-cre ${ }^{+}$ FSCs in $\mathrm{Ccl} 19^{\mathrm{EYFP} / \mathrm{iDTR}}$ mice led to a significantly reduced expansion of bgal ${ }_{96}$-specific $\mathrm{CD} 8^{+} \mathrm{T}$ cells in blood and lungs on day 21 when compared to those of PBS-treated controls (Fig. $4 \mathrm{~b}$ and Extended Data Fig. 4e). Likewise, the local depletion of EYFP ${ }^{+}$FSCs in the lung through i.n. DT application reduced $\mathrm{CD}^{+} \mathrm{T}$ cell inflation in blood and lungs (Fig. 4b and Extended Data Fig. 4e), suggesting that $\mathrm{Ccl19}-\mathrm{cre}^{+}$FSCs in the lung are the major cell population that drives the activation of inflating bgal $_{96}$-specific $\mathrm{CD}^{+} \mathrm{T}$ cells. The effect of local, lung-restricted depletion of $\mathrm{EYFP}^{+}$FSCs on expansion of inflating bgal ${ }_{96}$-specific $\mathrm{CD}^{+} \mathrm{T}$ cells became evident early after $\mathrm{T}$ cell activation (day 14) and was maintained until day 50 after immunization (Fig. 4c). Frequencies of bgal $_{497}$-specific CD8 ${ }^{+}$ $\mathrm{T}$ cells were not substantially altered by either of the ablation protocols (Extended Data Fig. 4f,g). $\mathrm{CD}^{+} \mathrm{T}$ cells in the lungs accumulated in the vicinity of pulmonary blood vessels, with single $\mathrm{CD}^{+} \mathrm{T}$ cells being in close contact with $\mathrm{EYFP}^{+}$FSCs in Ad-LacZ/ FlexON-vaccinated $C c l 19^{\text {EYFP/iDTR }}$ mice treated with PBS on day 21
(Fig. 4d, arrowheads). Such cellular aggregations harbored lymphocytes and macrophages leading to a significant increase in immune cell content compared to that of naive $C c l 19^{\text {EYFP/iDTR }}$ mice (Fig. $4 \mathrm{e}$ ). DT treatment (i.n.) reduced immune cell accumulation in lungs of Ccl1 $19^{\mathrm{EYFP} / \mathrm{iDTR}}$ mice with the most pronounced effect on $\mathrm{CD} 8^{+} \mathrm{T}$ cells (Fig. 4e and Extended Data Fig. 4h). To assess whether ablation of Ccl19-cre ${ }^{+}$FSCs in lungs affected the protective capacity of pulmonary immune cells, we challenged DT- or PBS-treated Ccl1 $9^{\mathrm{EYFP} / \mathrm{DDTR}}$ mice with bgal-expressing melanoma cells on day 21 after Ad-LacZ/ FlexON immunization (Fig. 4f). Mice with an intact lung FSC infrastructure controlled metastatic growth of tumor cells, whereas DT-treated mice were not protected and showed a significantly greater presence of tumor nodules in the lung (Fig. 4g,h). In sum, these data demonstrate that $\mathrm{Ccl} 19-\mathrm{cre}^{+}$FSCs not only substantially contribute to the expansion of inflationary memory $\mathrm{CD}^{+} \mathrm{T}$ cells that protect the host from metastasizing tumor but also transform the immune cell landscape in the lung in the long term.

Ad vectors reprogram $\mathrm{Ccl19-}$ - $r \boldsymbol{e}^{+}$lung fibroblasts. Although the number of Ccl19-cre ${ }^{+}$lung FSCs, highlighted by EYFP expression in $C c l 19-c r e ; R 26 R^{\mathrm{EYFP}}\left(\mathrm{Ccl1} 9^{\mathrm{EYFP}}\right)$ mice, was not increased on day 21 after Ad-LacZ/FlexON immunization (Fig. 5a and Extended Data Fig. 5a), cells showed a distinct activation pattern with enhanced surface expression of PDPN (Fig. 5b and Extended Data Fig. 5b), the adhesion molecule ICAM1 (Fig. $5 \mathrm{c}$ and Extended Data Fig. 5c) and platelet-derived growth factor receptor (PDGFR) $\alpha$ (CD140a) (Fig. 5d and Extended Data Fig. 5d). The expression of other canonical FSC markers such as PDGFR $\beta$ (Extended Data Fig. 5e) or the adhesion molecule VCAM1 (Extended Data Fig. 5f) was not substantially affected by Ad-LacZ/FlexON immunization. To elaborate in more detail molecular changes induced by the viral vector in pulmonary Ccl19-cre ${ }^{+}$cells, we took advantage of EYFP 
a
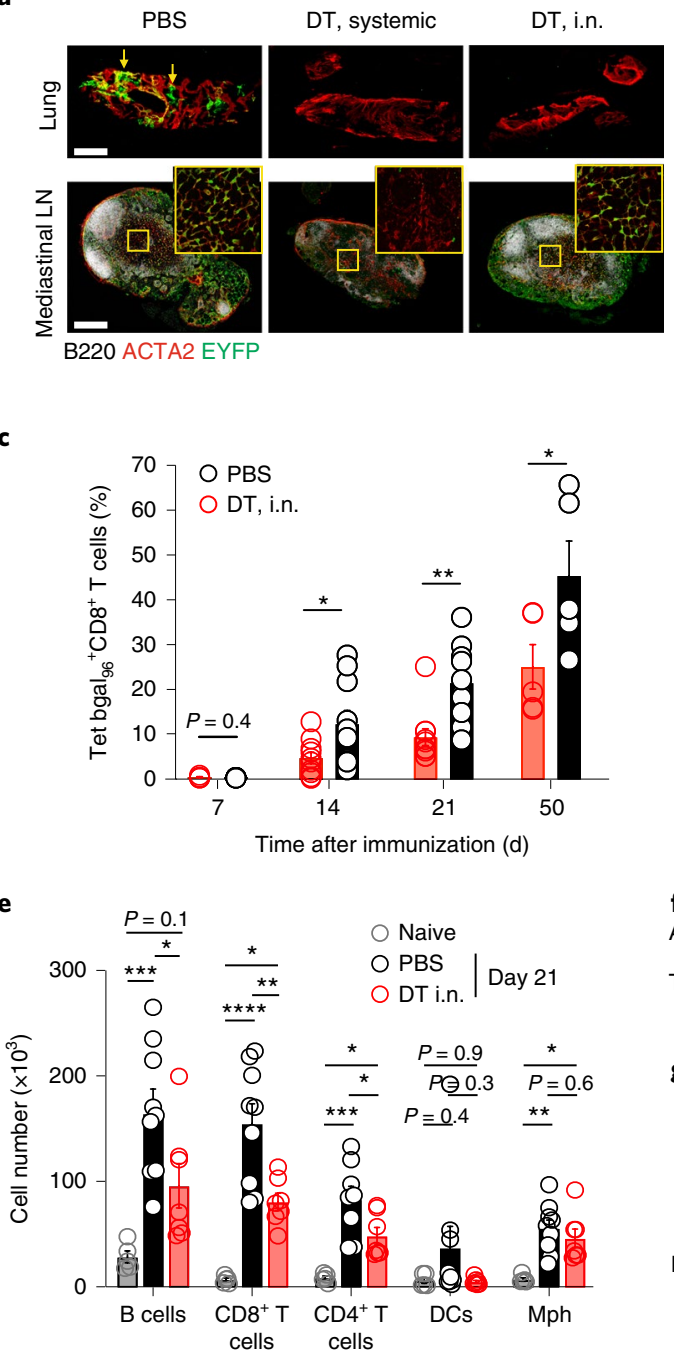

b

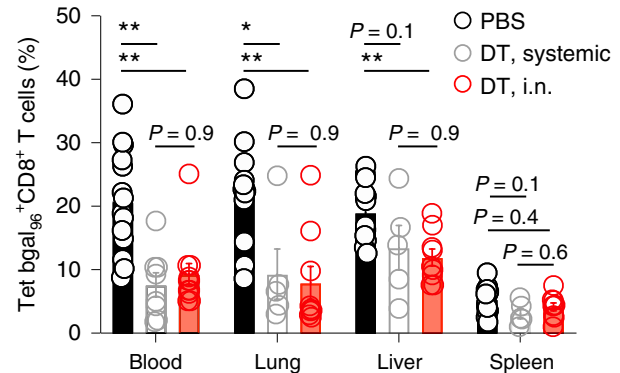

d

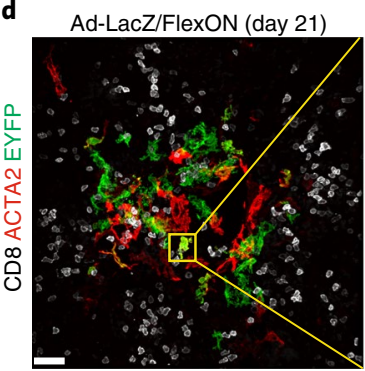

f

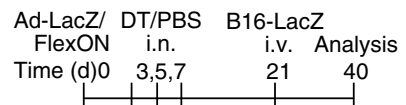

g

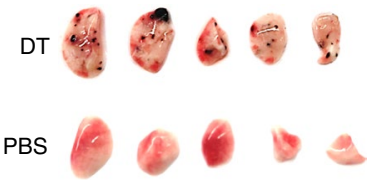

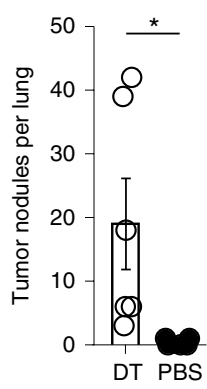

Fig. 4 | Pulmonary Ccl19-cre+ FSCs support inflating memory CD8+ T cells. a-e, Ccl19EYFP/DTR mice were i.v. immunized with Ad-LacZ/FlexON and treated i.n. or systemically with DT or PBS. a, Representative confocal microscopy images of EYFP+ FSCs in lungs and mediastinal lymph nodes (LN) after i.n. or systemic DT injection. Boxed and enlarged areas show the stromal network in the T cell zone of the lymph node. Scale bars, $150 \mu \mathrm{m}$ (overview) and $40 \mu \mathrm{m}$ (boxed areas). b. Frequency of bgal ${ }_{96}$ tetramer ${ }^{+} \mathrm{CD} 8^{+} \mathrm{T}$ cells in the indicated organs. $\mathbf{c}$, Frequency of bgal ${ }_{96}$ tetramer ${ }^{+} \mathrm{CD} 8^{+} \mathrm{T}$ cells in the blood at the indicated time points. d, Representative high-resolution immunofluorescence image of lungs from Ccl19 Arrowheads in the boxed areas indicate CD8 ${ }^{+} \mathrm{T}$ cells in close proximity to EYFP+ FSCs. Scale bar, $20 \mu \mathrm{m}$. e, Enumeration of immune cell populations in the lungs on day 21 in Ad-LacZ/FlexON-vaccinated mice following i.n. DT or PBS application or in naive mice; gating strategy is shown in Extended Data Fig. 4h. DCs, dendritic cells; Mph, macrophages. f, Treatment scheme for Ccl19EYFP/iDTR mice immunized with Ad-LacZ/FlexON and challenged i.v. with LacZ-expressing B16F10 melanoma cells. g,h, Analysis of lungs for pulmonary tumor metastases with representative images (g) and quantification (h). Values indicate mean \pm s.e.m. for each time point or organ analyzed. Pooled data from two independent experiments with $n=3$ (DT, systemic), $n=7$ (DT, i.n.) and $n=5$ (PBS control) mice (a); $n=5$ ( 8 for blood) (DT, systemic), $n=9$ (11 for blood) (DT, i.n.) and $n=14$ (PBS control) mice (b); $n=5$ (days 7 and 50), $n=10$ mice (day 14) and $n=8$ (day 21) mice (c); $n=3$ mice (d); $n=5$ (naive), $n=9$ (PBS, day 21) and $n=7$ (DT, i.n., day 21) mice (e); and $n=6$ mice $(\mathbf{g}, \mathbf{h})$. Statistical analysis was performed using one-way ANOVA with Tukey's multiple-comparisons test (b,e) and using unpaired two-tailed Student's $t$-test $(\mathbf{c}, \mathbf{h}) .{ }^{\star} P<0.05,{ }^{\star \star} P<0.01,{ }^{\star \star \star} P<0.001,{ }^{\star \star \star \star} P<0.0001$. Exact $P$ values are provided in the source data.

expression in $\mathrm{Ccl} 19^{\mathrm{EYFP}}$ mice to enrich cells and to analyze the transcriptome from naive and Ad-LacZ/FlexON-immunized mice by single-cell RNA sequencing (scRNA-seq). Unsupervised clustering of the combined samples defined six conserved clusters visualized with uniform manifold approximation and projection (UMAP; Fig. 5e,f). Computation of cluster-specific genes combined with analysis of known lung fibroblast signatures ${ }^{37-39}$ revealed transcriptional signatures consistent with two clusters of pulmonary fibroblasts (designated as $A q p 1^{\text {hi }}$ and $N p n t^{\mathrm{hi}}$ ) (Extended Data Fig. 6a), which form major fractions of EYFP ${ }^{+}$cells from both naive and immunized lungs (Fig. 5f). Vascular smooth muscle cells, characterized by the expression of Acta2, Tagln and Myh11, could be distinguished from pericytes, which are marked by the expression of Cox4i2, Postn and Notch3 (Extended Data Fig. 6a). The molecular signature of the third steady-state fibroblast fraction was distinguished by high-level expression of Lum (Fig. 5f and Extended Data Fig. 6a). Ad immunization induced the appearance of a molecularly distinct fraction with high expression levels of Il33, encoding a cytokine (Fig. 5e,f and Extended Data Fig. 6a). Il33 ${ }^{\text {hi }}$ fibroblasts appeared to be related to $\mathrm{Lum}^{\text {hi }}$ fibroblasts, as revealed by cluster similarity analysis (Fig. 5g). Using RNA velocity analysis of single cells, which provides a predictive value for cellular state progression 


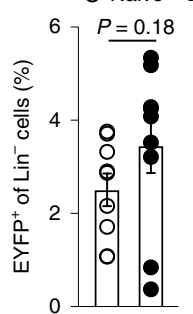

Ad-LacZ/FlexON (day 21)

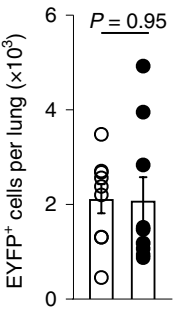

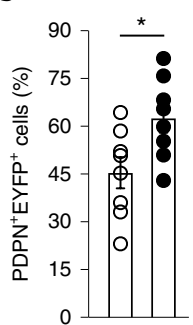

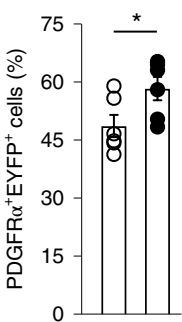

d

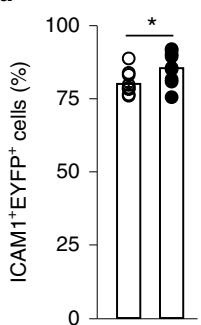

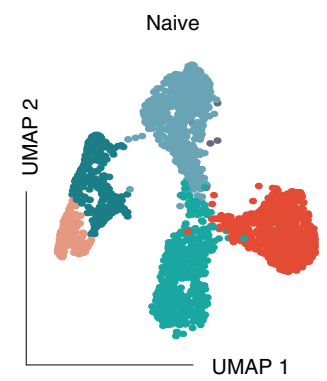

f

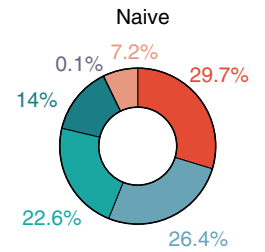

Ad-LacZ/FlexON (day 21)

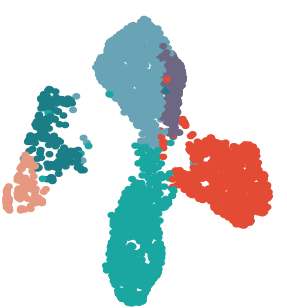

Ad-LacZ/FlexON (day 21)

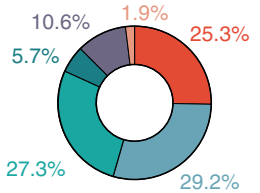

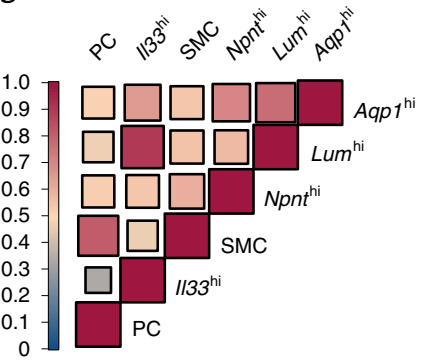

h

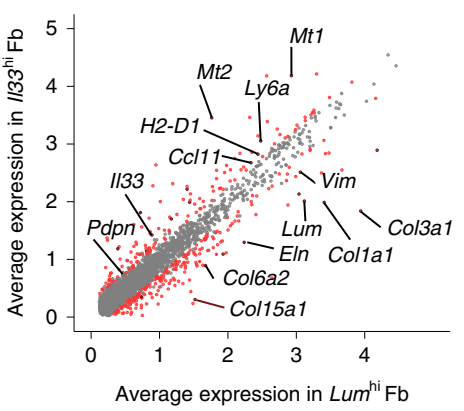

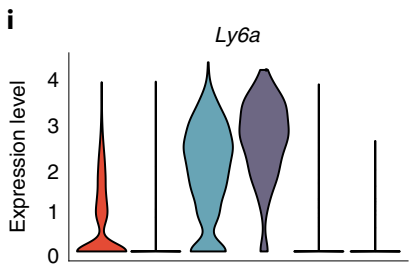

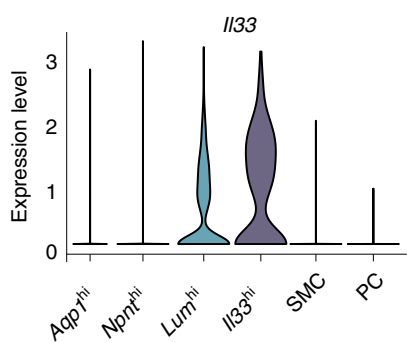

j
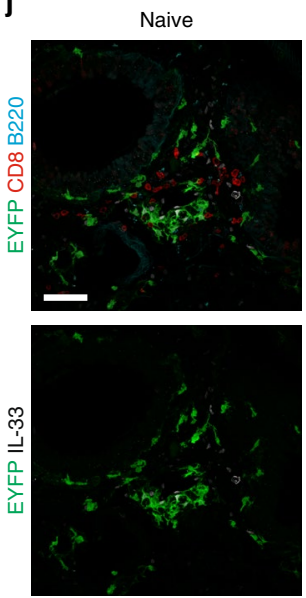

Ad-LacZ/FlexON (day 21)
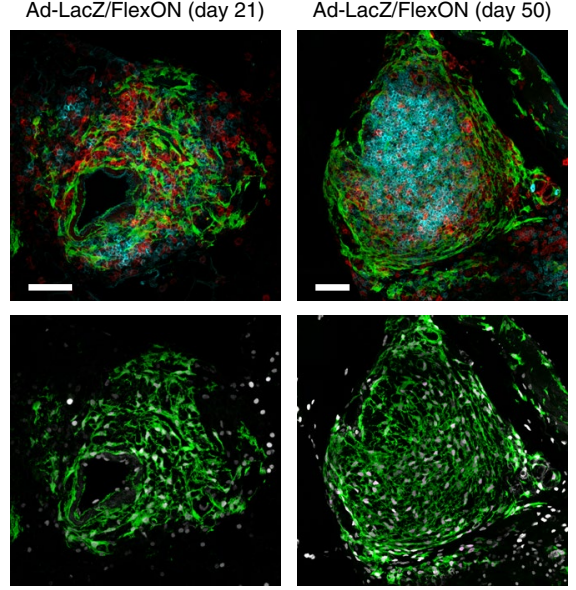

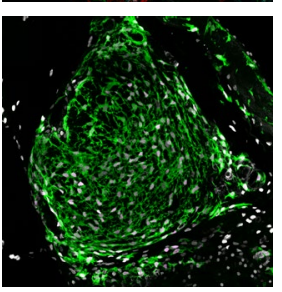

Fig. 5 | Ad-LacZ/FlexON activates pulmonary Ccl19-expressing, immune-stimulatory FSCs. Cc/19 ${ }^{\mathrm{EYFP}}$ mice were immunized i.v. with Ad-LacZ/FlexON, and pulmonary stromal cells were analyzed on day 21. a, Frequency (left) and absolute numbers of recovered EYFP+ cells (right). b-d, Frequency of PDPN+ (b), PDGFR $\alpha^{+}$(c) and ICAM1+ (d) cells within the EYFP+ population. e-i, sCRNA-seq analysis of EYFP+ cells isolated from lungs of Ccl19EYFP mice treated i.v. with Ad-LacZ/FlexON on day 21 or naive Ccl19 ${ }^{\mathrm{EYFP}}$ mice. Gating strategy is shown in Extended Data Fig. 5a. e, UMAP plots indicate FSC cluster assignment in naive control mice or Ad-LacZ/FlexON-treated mice. Fb, fibroblast. f, Pie charts displaying relative abundance of the identified pulmonary FSC clusters. g, Correlation plot of EYFP+ pulmonary FSCs depicting similarity between identified FSC clusters calculated as Pearson correlation based on the average gene expression. PC, pericyte; SMC, smooth muscle cell. h, Scatterplot showing average gene expression of $/ 133^{\text {hi }}$ FSCs versus $L$ um ${ }^{\text {hi }}$ FSCs in merged data from naive control mice and Ad-LacZ/FlexON-treated mice. i, Violin plots showing expression of II33 and Ly6a. j, Representative confocal microscopy images of BALT formation in the lungs of immunized Ccl19 ${ }^{\mathrm{EYFP}}$ mice on days 21 and 50 . Scale bars, $30 \mu \mathrm{m}$. scRNA-seq analysis was performed with two biological replicates for $n=6$ naive Cc/19-cre ${ }^{\mathrm{EYFP}}$ mice and $n=5$ Ad-LacZ/FlexON-treated mice. We obtained 2,465 (naive) and 2,891 (Ad-LacZ/FlexON) EYFP-expressing cells. Dots in a-d represent individual mice, and mean \pm s.e.m. are shown. Pooled data from two independent experiments with $n=9$ mice (a), $n=8$ mice (b), $n=6$ mice (c) and $n=9$ mice (d). j, Representative images from two independent experiments, $n=4$ mice per group. Statistical analysis was performed using unpaired two-tailed Student's $t$-test (a-d) with ${ }^{\star} P<0.05$. Exact $P$ values are provided in the source data. 
based on the balance between unspliced and spliced $\mathrm{mRNA}^{40}$, we found that Ad vector immunization induced dynamic changes in smooth muscle cell and pericyte compartments (Extended Data Fig. 6c). Likewise, the $I l 33^{\text {hi }}$ cell fraction showed highly dynamic gene expression, suggesting an ongoing differentiation processes, while the related $\mathrm{Lum}^{\mathrm{hi}}$ population was rather static (Extended Data Fig. 6c). Differential gene expression analysis between the two cell fractions showed that the Il $33^{\text {hi }}$ fibroblast population exhibits more immune-stimulatory functions, with upregulation of Il33 and Ly6a expression (Fig. 5h,i), and suggested that Ad immunization transforms the $\mathrm{Lum}^{\text {hi }}$ population from a structural, extracellular matrix-producing population to a metabolically active and immune-stimulating cell fraction (Extended Data Fig. 6b). This interpretation was supported by flow cytometric validation of the increased abundance of stem cell antigen 1 (Sca-1, encoded by the Ly6a gene)- and CD34-expressing EYFP $^{+}$fibroblasts (Extended Data Fig. 6d,e) and by PCR with reverse transcription (RT-PCR) analysis showing increased expression of Il33 in $\mathrm{EYFP}^{+}$lung cells of Ad-vaccinated mice compared to that of naive mice (Extended Data Fig. 6f). Moreover, the Sca- $1^{+} \mathrm{CD} 34^{+}$fraction of EYFP-expressing cells in lungs of Ad-vaccinated mice showed increased expression of Il33 mRNA compared to $\mathrm{EYFP}^{+} \mathrm{Sca}-1^{-}$cells (Extended Data Fig. 6g). The elevated expression of lacZ mRNA in the Sca- $1^{+} \mathrm{CD} 34^{+}$fraction of EYFP-expressing cells suggests that differentiation of $\mathrm{Lum}^{\mathrm{hi}}$ cells into immune-stimulatory FSCs is driven, at least partially, by the persistence Ad vector-delivered antigen in these cells (Extended Data Fig. 6h). Confocal microscopy analysis of thick lung sections revealed that $\mathrm{EYFP}^{+}$cells underpin mainly perivascular and peribronchial areas and that Ad vector immunization is associated with the formation of pronounced bronchus-associated lymphoid tissues (BALT) (Extended Data Fig. 7). We found aggregations of $\mathrm{CD}^{+}$ $\mathrm{T}$ cells enmeshed in a reticular network of IL-33+EYFP ${ }^{+}$FSCs on day 21 after Ad immunization (Fig. 5j and Extended Data Fig. 6i). By day 50 after immunization, Ccl19-cre $e^{+}$FSC-underpinned, highly organized BALT structures had developed, forming clearly distinguished areas of $\mathrm{B} 220^{+} \mathrm{B}$ cells surrounded by $\mathrm{CD} 8^{+} \mathrm{T}$ cells (Fig. 5 and Extended Data Fig. 6i). Overall, these results show that Ad immunization induces phenotypic and functional reprogramming of a distinct subset of lung fibroblasts, leading to elaboration of immune-dedicated niches that support maintenance of memory $\mathrm{CD}^{+} \mathrm{T}$ cells in the lung.

Ccl19-cre ${ }^{+}$FSCs maintain inflationary $\mathrm{CD8}^{+} \mathrm{T}$ cell fitness. To further elaborate how and to what extent local immune cell-nurturing fibroblastic niches support the maintenance and function of inflating memory $\mathrm{CD}^{+} \mathrm{T}$ cells, we used unbiased single-cell transcriptomics to gauge the phenotype of bgal $_{96}$-specific $\mathrm{CD} 8^{+} \mathrm{T}$ cells from the lungs of Ad-LacZ/FlexON-immunized $C c l 19^{\text {EYFP/iDTR }}$ mice. We found that the absence of Ccl19-cre ${ }^{+}$FSCs in DT-treated mice substantially changed the transcriptome of the majority of bgal $_{96}$-specific CD ${ }^{+} \mathrm{T}$ cells on day 21 in DT-treated mice compared with that of PBS-treated controls (Fig. 6a). Gene set enrichment and pathway analyses revealed that bgal $_{96}$-specific $\mathrm{CD} 8^{+} \mathrm{T}$ cells from the lungs of DT-treated mice switched to different metabolic circuits when $\mathrm{Ccl} 19-\mathrm{cre}^{+} \mathrm{FSCs}$ were absent. The presence of $\mathrm{Ccl19}-\mathrm{cre}^{+}$ FSCs in lungs of Ad-LacZ/FlexON-immunized (PBS-treated) mice led to the preservation of processes involved in the regulation of mitochondrial protein complexes including the electron transport chain and mitochondrial import, chromatin remodelers and the regulation of organelle membranes including endoplasmic reticulum-related trafficking (Fig. 6b and Extended Data Fig. 8a). By contrast, bgal ${ }_{96}$-specific $\mathrm{CD}^{+} \mathrm{T}$ cells deprived of $\mathrm{Ccl19}-\mathrm{cre}^{+}$ FSC niches in the lung activated metabolic processes involved in the regulation of ribosome activity and mitochondrial ATP synthase activity (Fig. 6b and Extended Data Fig. 8a). More detailed analysis of differentially expressed genes confirmed the decreased expression of genes involved in mitochondrial energy metabolism (for example, Cox5a, Cox8a, Ndufab1, Tomm20) in bgal ${ }_{96}$-specific $\mathrm{CD}^{+} \mathrm{T}$ cells from the DT-treated group (Fig. $6 \mathrm{c}$ and Extended Data Fig. 8b). Memory CD8 ${ }^{+} \mathrm{T}$ cells exhibit an increased mitochondrial mass that allows for improved energy provision via oxidative phosphorylation, which is required to maintain the functionality and survival of an antigen-experienced $\mathrm{T}$ cell population ${ }^{41,42}$. Although proliferation (Extended Data Fig. 8c) and survival (Extended Data Fig. 8d) were not affected by depletion of $\mathrm{Ccl19-cre}{ }^{+}$FSCs, analysis of the mitochondrial content of bgal $_{96}$-specific $\mathrm{CD}^{+} \mathrm{T}$ cells isolated from DT-treated $\mathrm{Ccl} 19^{\mathrm{EYFP} / \mathrm{iDTR}}$ mice revealed significantly lower mitochondrial mass (Fig. 6d) and mitochondrial membrane potential (Fig. 6e) compared to those of PBS-treated mice.

The appearance of an Il33 ${ }^{\text {hi }}$ FSC population in the lung following Ad immunization (Fig. 5) stimulated us to cross $I l 33^{\mathrm{t} / \mathrm{fl}}$ mice with Ccl19-cre mice. We found that the effect of cell-type-specific ablation of Il33 gene expression on the mitochondrial phenotype of bgal $_{96}$-specific CD8 ${ }^{+} \mathrm{T}$ cells was similar to that of DT-treated Ccl19 ${ }^{\text {EYFP } / \text { iDTR }}$ mice. Namely, mitochondrial mass (Fig. 6f,g), mitochondrial membrane potential (Fig. 6h) and the expression of mRNA coding for elements of the electron transport chain (Uqcrc2, cytochrome b-c1 complex subunit 2; Sdha, succinate dehydrogenase complex, subunit A; Cox4i1, cytochrome c oxidase subunit 4 isoform 1) were significantly lower in Ccl19-cre; $I l 33^{\mathrm{t} / \mathrm{fl}}$ mice than those in Ccl19-cre;Il33 ${ }^{+/+}$mice (Extended Data Fig. 8e). Moreover, Il33 gene deficiency in $\mathrm{Ccl19}-\mathrm{cre}^{+}$cells resulted in impaired expansion of bgal $_{96}$-specific $\mathrm{CD}^{+} \mathrm{T}$ cells on day 21 after immunization with Ad-LacZ/FlexON (Fig. 6i and Extended Data Fig. 9a). Of note, IL-33 deficiency in Ccl19-cre ${ }^{+}$cells and local DT-mediated ablation of $\mathrm{Ccl19}-\mathrm{cre}^{+}$lung FSCs impaired memory $\mathrm{CD}^{+} \mathrm{T}$ cell expansion to a similar degree (Fig. 6i and Extended Data Fig. 9a). Moreover, differentiation toward the effector memory phenotype with high expression of the markers KLRG1 and CX3CR1 (Fig. 6j) and the production of T cell effector cytokines (Fig. 6k,1) were affected to a similar extent by IL-33 deficiency and $\mathrm{Ccl1}$ - - cre $^{+}$lung FSC ablation. The expansion of bgal ${ }_{497}$-specific cells (Extended Data Fig. 9b), their differentiation (Extended Data Fig. 9c) and the production of T cell effector cytokines (Extended Data Fig. 9d) on day 21 after Ad-LacZ/ FlexON immunization was not affected by local Ccl19-cre ${ }^{+}$FSC ablation or cell-type-specific ablation of Il33 gene expression. In sum, these data reveal that IL-33 produced by $\mathrm{Ccl19}-\mathrm{cre}^{+}$FSCs plays a dominant role in generating pulmonary fibroblastic niches that are critical for the maintenance of the function and metabolic fitness of inflating memory $\mathrm{CD}^{+} \mathrm{T}$ cells.

\section{Discussion}

Ad vectors are a versatile vaccine platform as shown by the rapid development and clinical implementation of various formulations during the recent SARS-CoV-2 pandemic ${ }^{3,6,9,43,44}$. One of the prominent characteristics of Ad vectors, which is likely important for the generation of efficient cellular immune responses, is the persistence of the delivered antigen. In this study, we show that human Ad5-based vectors delivered via the i.v. route target lung FSCs and generate antigen depots in these long-lived cells. This feature is shown through a genetic approach that restricts antigen expression to specific cell types. The combination of limited antigen expression in $\mathrm{Ccl19}-\mathrm{cre}^{+}$ FSCs with impaired cross-presentation in bone marrow chimeric mice demonstrates that transfer of antigen to DCs is required for priming and the subsequent expansion of inflating memory $\mathrm{CD}^{+}$ $\mathrm{T}$ cells pools. In addition, the persistence of Ad vectors in lung tissue precipitated a profound remodeling of the FSC landscape that was associated with immune cell retention and the establishment of immune cell clusters mainly in perivascular and peribronchial niches.

Sustained antigen presentation in the local target tissue within the critical fibroblastic niche explains many of the known distinctive traits of $\mathrm{CD}^{+} \mathrm{T}$ cell responses generated by Ad vectors that underpin 


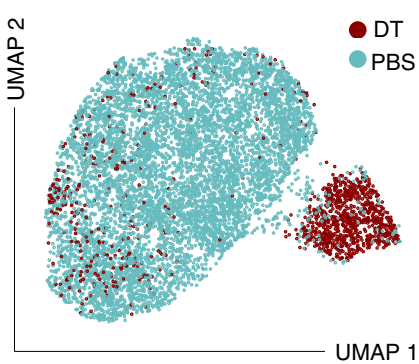

b

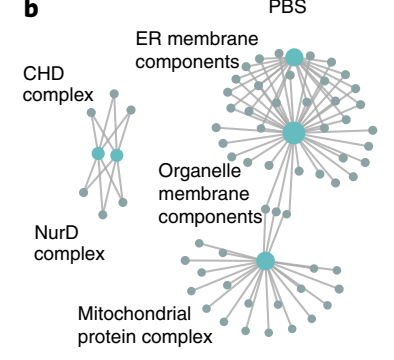

DT

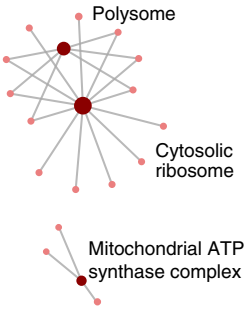

c

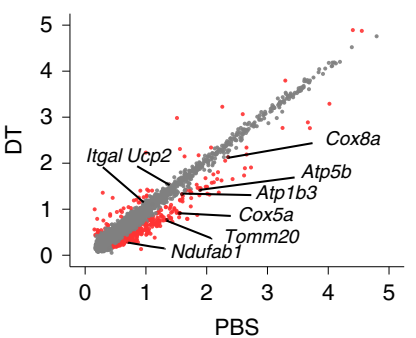

d

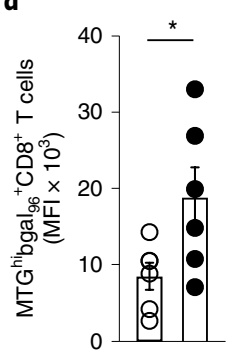

e

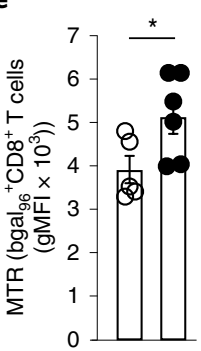

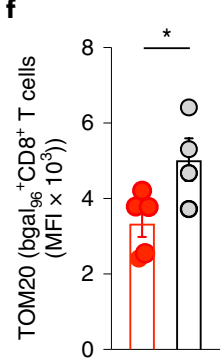

g

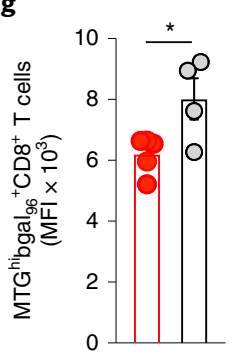

h

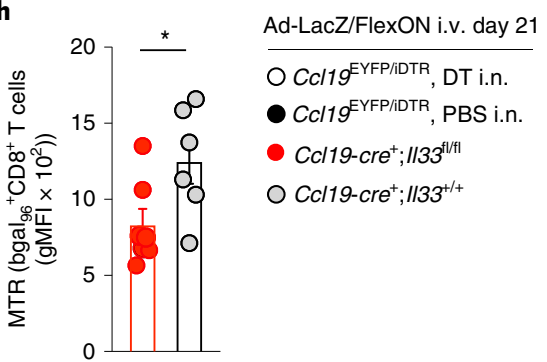

i

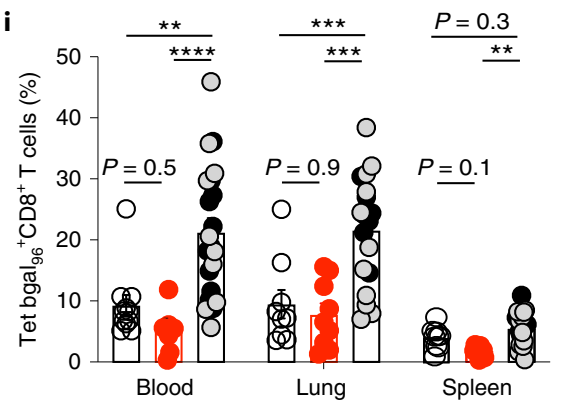

j

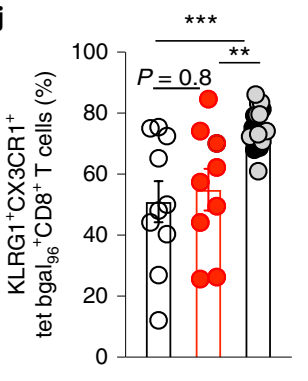

k

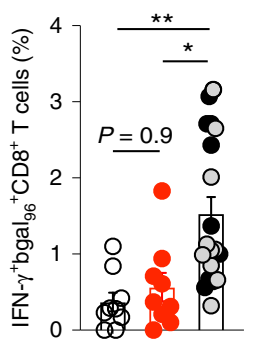

I

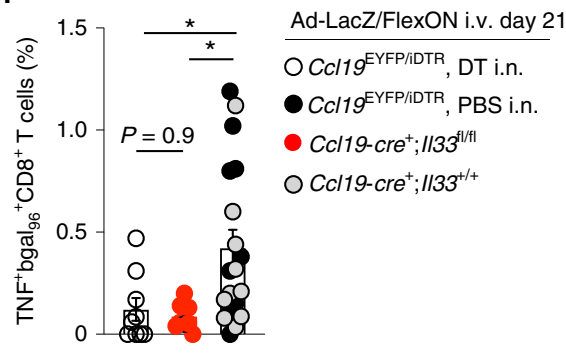

Fig. 6 | Pulmonary FSC-derived IL-33 preserves metabolic and functional fitness of inflationary memory CD8 ${ }^{+}$T cells. a-e, CCl19EYFP/IDTR mice were

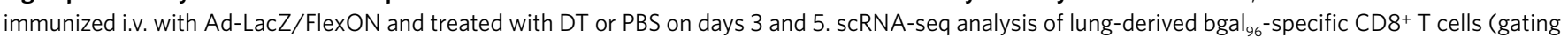
strategy is shown in Extended Data Fig. 2d). a, UMAP plots of bgal ${ }_{96}$-specific CD8+ T cells. $\mathbf{b}$, Network plots displaying most significantly enriched gene ontologies and the number of enriched genes based on transcriptional differences in bgal ${ }_{96}{ }^{-s p e c i f i c ~ C D 8}{ }^{+} \mathrm{T}$ cells. ER, endoplasmic reticulum. $\mathbf{c}$, Scatterplot displaying differentially expressed genes in bgal ${ }_{96}$-specific CD8+ T cells. d,e, Mean expression of MitoTracker Green (MTG) in the MTG-high population $\left(\mathrm{MTG}^{\text {hi }}\right.$ ) (d) and the geometric mean of MitoTracker Red (MTR) signal (e) in pulmonary bgal ${ }_{96}$-specific T cells. MFI, mean fluorescence intensity; gMFI,

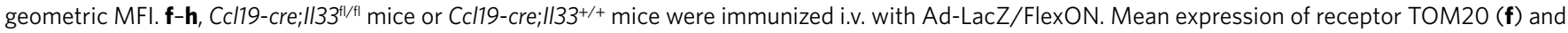
MTG in the MTG ${ }^{\text {hi }}$ fraction ( $\mathbf{g}$ ) and the geometric mean of MTR signal (h) in pulmonary bgal ${ }_{96}$-specific T cells. $\mathbf{i}-\mathbf{I}$, CD8 ${ }^{+} \mathrm{T}$ cells were analyzed $21 \mathrm{~d}$ after

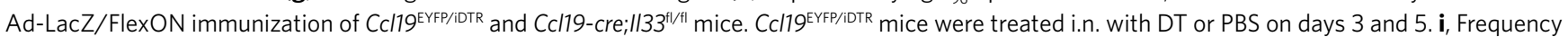
of bgal ${ }_{96}$-specific T cells in blood, lungs and spleen. $\mathbf{j}$, Frequency of KLRG1+CX3CR $1^{+}$bgal ${ }_{96}$-specific $T$ cells in the lungs. $\mathbf{k}, \mathbf{I}$, Interferon (IFN)- $\gamma$ - and tumor necrosis factor (TNF)-producing pulmonary bgal ${ }_{96}$-specific CD8+ T cells. Dots represent individual mice. Bar graphs indicate means, and lines show \pm s.e.m. scRNA-seq analysis was performed with two biological replicates for $n=5$ PBS-treated and $n=6$ DT-treated, Ad-LacZ/FlexON-immunized,

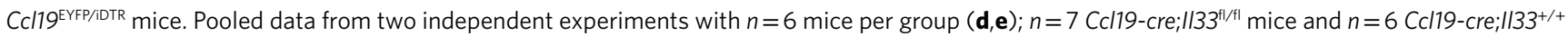

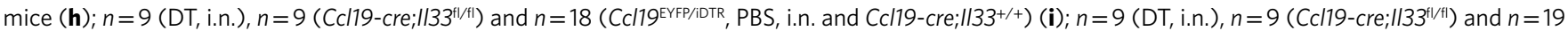

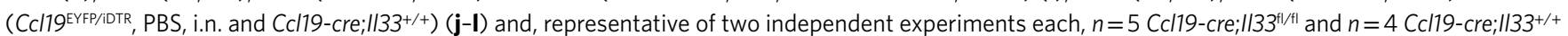
mice $(\mathbf{f}, \mathbf{g})$. Statistical analysis was conducted using unpaired two-tailed Student's t-test (d-h) or one-way ANOVA with Tukey's multiple-comparisons test (i-I) with ${ }^{\star} P<0.05,{ }^{\star \star} P<0.01,{ }^{\star \star \star} P<0.001,{ }^{\star \star \star \star} P<0.0001$. Exact $P$ values are provided in the source data.

the high protective capacity of this vaccine platform. The phenotypic and functional traits of these cells, including the transcriptional profile, are well reproduced in human studies of CMV and importantly of adenoviral vector immunization, including chimpanzee-derived vectors as used for coronavirus disease 2019 (refs. ${ }^{6,45,46}$ ). However, there are more features to explain. Our data provide evidence that local provision of IL-33 by Ccl19-cre ${ }^{+}$FSCs in the lung is critical for the inflationary expansion of memory $\mathrm{CD}^{+} \mathrm{T}$ cells and their function. Due to rapid conformational changes induced by the binding to its receptor, IL-33 only has a short range of action ${ }^{47}$. Hence, it is likely that the effect of IL-33 generated by Ccl19-cre $e^{+}$FSCs is limited to the tissue and the particular microenvironmental niche where the alarmin is produced. IL-33 can activate multiple cell types expressing the IL-33 receptor (ST2, IL1RL1), including stromal niche cells to produce cytokines supporting immune cell expansion ${ }^{48}$ and DCs to mature and cross-present antigen ${ }^{49}$. It is thus conceivable that FSC-derived IL-33 stimulates both cross-presenting DCs and antigen-specific $\mathrm{CD}^{+} \mathrm{T}$ cells in the particular niche environment. Indeed, IL-33 can directly stimulate T cells during acute viral infection $^{50}$ for the efficient generation of a memory-recall response ${ }^{51}$. Of note, provision of exogenous IL-33 was shown to augment inflating memory $\mathrm{CD}^{+} \mathrm{T}$ cell responses during MCMV infection, whereas the $\mathrm{T}$ cell priming phase was not dependent on the adjuvant effect of the cytokine ${ }^{52}$. It is possible that the adjuvant effect of IL-33 
on inflating memory $\mathrm{CD}^{+} \mathrm{T}$ cells depends, to some extent, on metabolic pathways that support the acquisition of an optimal energetic state and thereby fosters survival in peripheral tissue. Based on our data, it is conceivable that IL-33 promotes mitochondrial function by increasing mitochondrial mass and membrane polarization. It will be important in future studies to determine whether inflationary memory $\mathrm{CD}^{+} \mathrm{T}$ cells are the major, or even exclusive, target cell of IL-33 in immunostimulatory niches generated by Ad vector vaccination and whether modulation of metabolic pathways could further sustain the fitness of memory $\mathrm{T}$ cells in the particular tissue context.

Immunostimulatory FSCs of secondary lymphoid organs, known as FRCs, generate distinct niches for immune cell activation, differentiation and sustenance ${ }^{21,22}$. For example, lymph node FRC subsets underpinning the B cell area, that is, CXCL13-expressing follicular DCs regulate the germinal center reaction ${ }^{53}$, while marginal reticular cells provide niches for $\mathrm{CD}_{169^{+}}$sinusoidal macrophages through the provision of the cytokine RANKL ${ }^{54}$. Although the lung is not a dedicated lymphoid organ, particular areas in the lung can develop features of lymphoid organs that are described as inducible BALT $^{55}$. Pulmonary immune cell aggregations induced by intravenous Ad vector immunization were mainly located in the vicinity of mid-sized ACTA2 ${ }^{+}$blood vessels in the peribronchial interstitium of the hilus region. As both lacZ DNA and mRNA can be detected for more than $100 \mathrm{~d}$ after Ad5-LacZ immunization ${ }^{18}$, it is likely that Ad vector particles injected into the venous bloodstream are filtered out in the vascular system of the lung, leading to transduction of FSCs in the perivascular space. Moreover, it is possible that the persistence of Ad vectors in lung FSCs provides both innate activation signals and mediates antigen-dependent contact with lymphocytes leading to the formation of FSC-immune cell aggregates in the perivascular-peribronchial space.

The perivascular fibroblast compartment in the lung was shown to support $\mathrm{CD}^{+} \mathrm{T}$ cell activity against tumor cells ${ }^{33}$ and to regulate the activity of type 2 innate lymphoid cells ${ }^{56}$. Molofsky and colleagues $^{56}$ demonstrated that such adventitial niches formed by IL-33-producing fibroblasts exist in several tissues and that these circuits are important for the long-term control of helminth infection. Hence, immunostimulatory FSCs contribute to the formation of critical perivascular niches in different tissues to maintain protective $\mathrm{T}$ cell responses.

In sum, the approach of Ad vector-mediated generation of local antigenic depots as we have demonstrated can generate very substantial local immunity in the lung. Creating such depots in long-lived fibroblasts together with the reprogramming of fibroblastic niches to sustain local T cell responses could be relevant in development of local immunity. Moreover, such local immune-stimulatory niches could contribute to systemic protection as evidenced by the widespread distribution of $\mathrm{T}$ cells following Ad vector vaccination. In the context of global pandemics, it is of clear relevance to further explore and potentially exploit the ability of Ad vectors and other vaccine platforms to specifically reprogram FSCs in different tissues. Research on this particular pathway for immunization against emerging pathogens in both preclinical models and in clinical studies could help to further accelerate vaccine development.

\section{Online content}

Any methods, additional references, Nature Research reporting summaries, source data, extended data, supplementary information, acknowledgements, peer review information; details of author contributions and competing interests; and statements of data and code availability are available at https://doi.org/10.1038/ s41590-021-00969-3.

Received: 1 October 2020; Accepted: 7 June 2021; Published online: 15 July 2021

\section{References}

1. Chang, J. T., Wherry, E. J. \& Goldrath, A. W. Molecular regulation of effector and memory T cell differentiation. Nat. Immunol. 15, 1104-1115 (2014).

2. Priddy, F. H. et al. Safety and immunogenicity of a replication-incompetent adenovirus type $5 \mathrm{HIV}-1$ clade $\mathrm{B} \mathrm{gag} / \mathrm{pol} / \mathrm{nef}$ vaccine in healthy adults. Clin. Infect. Dis. 46, 1769-1781 (2008).

3. Stephenson, K. E. et al. Immunogenicity of the Ad26.COV2.S vaccine for COVID-19. JAMA 325, 1535-1544 (2021).

4. Snook, A. E. et al. Split tolerance permits safe Ad5-GUCY2C-PADRE vaccine-induced T-cell responses in colon cancer patients. J. Immunother. Cancer 7, 104 (2019).

5. Ewer, K. et al. Chimpanzee adenoviral vectors as vaccines for outbreak pathogens. Hum. Vaccin. Immunother. 13, 3020-3032 (2017).

6. Folegatti, P. M. et al. Safety and immunogenicity of the ChAdOx1 nCoV-19 vaccine against SARS-CoV-2: a preliminary report of a phase $1 / 2$, single-blind, randomised controlled trial. Lancet 396, 467-478 (2020).

7. Ewer, K. J. et al. T cell and antibody responses induced by a single dose of ChAdOx1 nCoV-19 (AZD1222) vaccine in a phase 1/2 clinical trial. Nat. Med. 27, 270-278 (2021).

8. Barnes, E. et al. Novel adenovirus-based vaccines induce broad and sustained T cell responses to HCV in man. Sci. Transl. Med. 4, 115ral11 (2012).

9. Logunov, D. Y. et al. Safety and efficacy of an rAd26 and rAd5 vector-based heterologous prime-boost COVID-19 vaccine: an interim analysis of a randomised controlled phase 3 trial in Russia. Lancet 397, 671-681 (2021).

10. Klenerman, P. The (gradual) rise of memory inflation. Immunol. Rev. 283, 99-112 (2018)

11. Karrer, U. et al. Memory inflation: continuous accumulation of antiviral $\mathrm{CD} 8^{+}$ T cells over time. J. Immunol. 170, 2022-2029 (2003).

12. Sierro, S., Rothkopf, R. \& Klenerman, P. Evolution of diverse antiviral CD8 ${ }^{+}$ $\mathrm{T}$ cell populations after murine cytomegalovirus infection. Eur. J. Immunol. 35, 1113-1123 (2005).

13. Holtappels, R., Pahl-Seibert, M. F., Thomas, D. \& Reddehase, M. J. Enrichment of immediate-early 1 ( $\mathrm{m} 123 / \mathrm{pp} 89)$ peptide-specific CD8 T cells in a pulmonary $\mathrm{CD}_{2} 2 \mathrm{~L}^{\mathrm{lo}}$ memory-effector cell pool during latent murine cytomegalovirus infection of the lungs. J. Virol. 74, 11495-11503 (2000).

14. Grzimek, N. K., Dreis, D., Schmalz, S. \& Reddehase, M. J. Random, asynchronous, and asymmetric transcriptional activity of enhancer-flanking major immediate-early genes $i e 1 / 3$ and $i e 2$ during murine cytomegalovirus latency in the lungs. J. Virol. 75, 2692-2705 (2001).

15. Snyder, C. M. et al. Memory inflation during chronic viral infection is maintained by continuous production of short-lived, functional $\mathrm{T}$ cells. Immunity 29, 650-659 (2008).

16. Komatsu, H., Sierro, S., Cuero, A. V. \& Klenerman, P. Population analysis of antiviral T cell responses using MHC class I-peptide tetramers. Clin. Exp. Immunol. 134, 9-12 (2003).

17. Klenerman, P. \& Oxenius, A. T cell responses to cytomegalovirus. Nat. Rev. Immunol. 16, 367-377 (2016).

18. Bolinger, B. et al. A new model for $\mathrm{CD}^{+} \mathrm{T}$ cell memory inflation based upon a recombinant adenoviral vector. J. Immunol. 190, 4162-4174 (2013).

19. Lee, L. N. et al. Adenoviral vaccine induction of $\mathrm{CD} 8^{+} \mathrm{T}$ cell memory inflation: impact of co-infection and infection order. PLoS Pathog. 13, e1006782 (2017)

20. Bassett, J. D. et al. CD8 ${ }^{+}$T-cell expansion and maintenance after recombinan adenovirus immunization rely upon cooperation between hematopoietic and nonhematopoietic antigen-presenting cells. Blood 117, 1146-1155 (2011).

21. Krishnamurty, A. T. \& Turley, S. J. Lymph node stromal cells: cartographers of the immune system. Nat. Immunol. 21, 369-380 (2020).

22. Perez-Shibayama, C., Gil-Cruz, C. \& Ludewig, B. Fibroblastic reticular cells at the nexus of innate and adaptive immune responses. Immunol. Rev. 289, 31-41 (2019).

23. Pikor, N. B., Cheng, H. W., Onder, L. \& Ludewig, B. Development and immunological function of lymph node stromal cells. J. Immunol. 206, 257-263 (2021).

24. Torti, N., Walton, S. M., Murphy, K. M. \& Oxenius, A. Batf3 transcription factor-dependent DC subsets in murine CMV infection: differential impact on T-cell priming and memory inflation. Eur. J. Immunol. 41, 2612-2618 (2011).

25. Torti, N., Walton, S. M., Brocker, T., Rülicke, T. \& Oxenius, A. Non-hematopoietic cells in lymph nodes drive memory CD8 T cell inflation during murine cytomegalovirus infection. PLoS Pathog. 7, e1002313 (2011).

26. Busche, A. et al. Priming of CD8 $8^{+} \mathrm{T}$ cells against cytomegalovirusencoded antigens is dominated by cross-presentation. J. Immunol. 190, 2767-2777 (2013).

27. Baumann, N. S. et al. Tissue maintenance of CMV-specific inflationary memory T cells by IL-15. PLoS Pathog. 14, e1006993 (2018).

28. Rowe, W. P. Studies on pathogenesis and immunity in lymphocytic choriomeningitis infection of the mouse. Navy Res. Rep. 12, 167-220 (1954).

29. Atasoy, D., Aponte, Y., Su, H. H. \& Sternson, S. M. A FLEX switch targets channelrhodopsin-2 to multiple cell types for imaging and long-range circuit mapping. J. Neurosci. 28, 7025-7030 (2008). 
30. Chai, Q. et al. Maturation of lymph node fibroblastic reticular cells from myofibroblastic precursors is critical for antiviral immunity. Immunity $\mathbf{3 8}$, 1013-1024 (2013).

31. Cheng, H. W. et al. Origin and differentiation trajectories of fibroblastic reticular cells in the splenic white pulp. Nat. Commun. 10, 1739 (2019).

32. Cupovic, J. et al. Central nervous system stromal cells control local CD8 ${ }^{+}$ $\mathrm{T}$ cell responses during virus-induced neuroinflammation. Immunity $\mathbf{4 4}$, 622-633 (2016).

33. Cheng, H. W. et al. CCL19-producing fibroblastic stromal cells restrain lung carcinoma growth by promoting local antitumor T-cell responses. J. Allergy Clin. Immunol. 142, 1257-1271 (2018).

34. Hildner, K. et al. Batf3 deficiency reveals a critical role for $\mathrm{CD} 8 \alpha^{+}$dendritic cells in cytotoxic T cell immunity. Science 322, 1097-1100 (2008).

35. Krebs, P., Scandella, E., Odermatt, B. \& Ludewig, B. Rapid functional exhaustion and deletion of CTL following immunization with recombinant adenovirus. J. Immunol. 174, 4559-4566 (2005).

36. Novkovic, M. et al. Topological small-world organization of the fibroblastic reticular cell network determines lymph node functionality. PLoS Biol. 14, e1002515 (2016)

37. Zepp, J. A. et al. Distinct mesenchymal lineages and niches promote epithelial self-renewal and myofibrogenesis in the lung. Cell 170, 1134-1148 (2017)

38. Xie, T. et al. Single-cell deconvolution of fibroblast heterogeneity in mouse pulmonary fibrosis. Cell Rep. 22, 3625-3640 (2018).

39. Tsukui, T. et al. Collagen-producing lung cell atlas identifies multiple subsets with distinct localization and relevance to fibrosis. Nat. Commun. 11, 1920 (2020).

40. La Manno, G. et al. RNA velocity of single cells. Nature 560, 494-498 (2018).

41. van der Windt, G. J. et al. Mitochondrial respiratory capacity is a critical regulator of $\mathrm{CD}^{+} \mathrm{T}$ cell memory development. Immunity 36, 68-78 (2012).

42. van der Windt, G. J. et al. CD8 memory T cells have a bioenergetic advantage that underlies their rapid recall ability. Proc. Natl Acad. Sci. USA 110 14336-14341 (2013).

43. Rego, G. N. A. et al. Current clinical trials protocols and the global effort for immunization against SARS-CoV-2. Vaccines 8, 474 (2020).
44. Mercado, N. B. et al. Single-shot Ad26 vaccine protects against SARS-CoV-2 in rhesus macaques. Nature 586, 583-588 (2020).

45. Bolinger, B. et al. Adenoviral vector vaccination induces a conserved program of $\mathrm{CD}^{+} \mathrm{T}$ cell memory differentiation in mouse and man. Cell Rep. 13, 1578-1588 (2015).

46. Gordon, C. L. et al. Induction and maintenance of CX3CR1-intermediate peripheral memory $\mathrm{CD}^{+} \mathrm{T}$ cells by persistent viruses and vaccines. Cell Rep. 23, 768-782 (2018).

47. Cohen, E. S. et al. Oxidation of the alarmin IL-33 regulates ST2-dependent inflammation. Nat. Commun. 6, 8327 (2015).

48. Mager, L. F. et al. IL-33 signaling contributes to the pathogenesis of myeloproliferative neoplasms. J. Clin. Invest. 125, 2579-2591 (2015).

49. Dominguez, D. et al. Exogenous IL-33 restores dendritic cell activation and maturation in established cancer. J. Immunol. 198, 1365-1375 (2017).

50. Bonilla, W. V. et al. The alarmin interleukin-33 drives protective antiviral $\mathrm{CD}^{+}$T cell responses. Science 335, 984-989 (2012).

51. Baumann, C. et al. Memory $\mathrm{CD}^{+} \mathrm{T}$ cell protection from viral reinfection depends on interleukin-33 alarmin signals. Front. Immunol. 10, 1833 (2019).

52. McLaren, J. E. et al. IL-33 augments virus-specific memory T cell inflation and potentiates the efficacy of an attenuated cytomegalovirus-based vaccine. J. Immunol. 202, 943-955 (2019).

53. Pikor, N. B. et al. Remodeling of light and dark zone follicular dendritic cells governs germinal center responses. Nat. Immunol. 21, 649-659 (2020).

54. Camara, A. et al. Lymph node mesenchymal and endothelial stromal cells cooperate via the RANK-RANKL cytokine axis to shape the sinusoidal macrophage niche. Immunity 50, 1467-1481 (2019).

55. Silva-Sanchez, A. \& Randall, T. D. Role of iBALT in respiratory immunity. Curr. Top. Microbiol. Immunol. 426, 21-43 (2020).

56. Dahlgren, M. W. et al. Adventitial stromal cells define group 2 innate lymphoid cell tissue niches. Immunity 50, 707-722 (2019).

Publisher's note Springer Nature remains neutral with regard to jurisdictional claims in published maps and institutional affiliations.

(c) The Author(s), under exclusive licence to Springer Nature America, Inc. 2021 


\section{Methods}

Mice. BAC transgenic C57BL/6N-Tg(Ccl19-cre)489Biat (Ccl19-cre) mice were previously described ${ }^{30}$. C57BL/6N (B6), C57BL/6N and R26R ${ }^{\mathrm{EYFP}}$ (B6.129X1-Gt ROSA)26Sor ${ }^{\text {tml(EYFP)Cos } / J) ~ m i c e ~ w e r e ~ p u r c h a s e d ~ f r o m ~ C h a r l e s ~ R i v e r, ~ a n d ~} L y s M$-cre mice were purchased from Jackson Laboratory. $\mathrm{H} 2-\mathrm{K}^{b-1-}$ mice were purchased from Taconic Farms. R26R ${ }^{\text {iDTR }}$ (C57BL/6-Gt(ROSA)26Sortml(HBEGF)Awai $/$ J) mice were obtained from A. Waisman (University of Mainz), and Cd11c-cre mice were obtained from B. Reizis (New York University School of Medicine) and Ubi-cre ${ }^{\text {ERT2 }}$ mice were obtained from T. Vincent (Kennedy Institute, University of Oxford). The Il33-targeting vector to generate $I l 33^{\mathrm{t} / / \mathrm{l}}$ mice was generated by the trans-NIH Knock-Out Mouse Project (KOMP, project ID CSD88909) and obtained from the KOMP Repository (https://www.komp.org/). To ablate Il33 expression in a distinct FSC subset, $\mathrm{Ccl19}$-cre mice were crossed with $\mathrm{Il} 33^{\mathrm{t} / \mathrm{l}} \mathrm{mice}$. Ccl19-cre mice were bred with $R 26 R^{\mathrm{EYFP}}$ or Rosa26 $2 R^{\mathrm{iDTR}}$ and $R 26 R^{\mathrm{EYFP}}$ mice to generate heterozygous $C c l 19-c r e ; R 26 R^{\mathrm{EYFP}}$ and $C c l 19-\mathrm{cre} ; R 26 R^{\mathrm{EYFP} / \mathrm{IDTR}}$ mice. Batf $3^{-1-}$ mice were obtained from M. Kopf (ETH Zurich). All mice were housed in the Institute of Immunobiology, Kantonsspital St. Gallen under specific-pathogen-free conditions at $22^{\circ} \mathrm{C}$ with $30-70 \%$ humidity in a $12-12 \mathrm{~h}$ light-dark cycle and provided ad libitum access to food and water. All experiments were performed with 7-10-week-old mice (males and females) in accordance with federal and cantonal guidelines (Tierschutzgesetz) under permission numbers SG08/17, SG01/18, SG07/19, SG04/20 and SG01/20 following review and approval by the respective cantonal veterinary offices (St. Gallen, Switzerland).

Generation of bone marrow chimeric mice. Recipient mice were lethally irradiated with $9 \mathrm{~Gy}$ from a linear accelerator (Clinic of Radio-Oncology, Kantonal Hospital, St. Gallen). Within $24 \mathrm{~h}$, recipients were injected with $1.5-3 \times 10^{7}$ donor BM cells obtained from C57BL/6N, $\mathrm{H}_{2-\mathrm{K}^{b-/}}$ or $\mathrm{Batf} 3^{-/-}$mice. Mice were maintained on antibiotic water containing sulfadoxin and trimethoprim (Borgal, Veterinaria) for 3 weeks. From 6 weeks after BM transplantation, chimeric mice were bled every week to assess repopulation of the hematopoietic compartment in blood. Once full hematopoietic compartment repopulation had been achieved (8-12 weeks after BM transplantation) mice were used in experiments.

Cell ablation. Local lung FSC ablation was achieved by i.n. applying DT ( $8 \mathrm{ng}$ in $10 \mu \mathrm{l}$ PBS) to $C c l 19-c r e ; R 26 R^{\mathrm{EYFP} / \mathrm{iDTR}}$ or $C c l 19-\mathrm{cre} ; \mathrm{R}_{26} \mathrm{R}^{\mathrm{EYFP}}$ control mice on days 3 and 5 (and day 7 for tumor protection experiments) after immunization with Ad-LacZ/FlexON. Flow cytometry was used to assess ablation efficiency, and lung draining lymph nodes were inspected for the integrity of the FRC network by confocal microscopy on day 7 after immunization. Systemic FSC ablation was achieved by giving intraperitoneal injections of DT (8 ng per g body weight $)^{36}$. Ablation efficiency was assessed by confocal microscopy and flow cytometry.

Generation of adenoviral vectors. The Ad-LacZ/FlexON vector was generated by Vector Biolabs. The vector is based on a viral backbone of the human Ad5 sequence that includes 5' L-ITR and packaging signal. This is followed by a Flex switch that uses the CMV promoter and carries the lacZ gene in inverted orientation followed by a polyA tail. The $3^{\prime}$ end of the construct is composed of human Ad5 sequences that carry deletions in $E 1$ and $E 3$ genes and ends with a $3^{\prime}$ R-ITR. The Flex switch ${ }^{29}$ uses two pairs of heterotypic, antiparallel loxP-type recombination sites, which first undergo an inversion of the coding sequence, followed by excision of two sites, leading to one of each orthogonal recombination site oppositely oriented and incapable of further recombination. Recombinant Ad expressing the bgal protein under the control of the HCMV promoter (Ad-LacZ) and lacking $E 1$ and $E 3$ genes was used as described previously ${ }^{18}$. Both vector preparations were applied i.v. at $1 \times 10^{8} \mathrm{IU}$ per mouse (all diluted in PBS in a volume of $200 \mu \mathrm{l}$ per mouse). Human AdHu5-GFP and chimpanzee ChAdOx1-GFP vectors were produced by the Jenner Institute Viral Vector Core Facility at the University of Oxford as previously described $^{5}$

Isolation of murine cells. Mice were killed at the indicated time points and immediately perfused with PBS. Lung-infiltrating lymphocytes were isolated using mechanical disruption of the organ. For isolation of myeloid and stromal cells from the lung, the tissue was cut into small pieces and transferred into a 24-well dish filled with RPMI 1640 medium containing 2\% FCS, 20 mM HEPES, pH 7.2 (all from Lonza), $1 \mathrm{mg} \mathrm{ml}^{-1}$ Collagenase Type P (Sigma-Aldrich), $25 \mu \mathrm{g} \mathrm{ml}^{-1}$ DNase I (AppliChem) and $1 \mathrm{mg} \mathrm{ml}^{-1}$ Collagenase Type II (Sigma-Aldrich) in combination with gentleMACS-based mechanical disruption (Miltenyi Biotec). After a 30-min incubation at $37^{\circ} \mathrm{C}$, cell suspensions were washed with PBS containing $0.5 \%$ FCS and $10 \mathrm{mmoll}^{-1}$ EDTA. Stromal cell fraction enrichment was achieved by depleting hematopoietic and erythroid cells using MACS anti-CD45 and anti-Ter119 microbeads (Miltenyi Biotec).

Cell lines. The murine B16 melanoma LacZ-expressing cell line (B16-LacZ) was obtained from RIKEN BRC (cell no. RCB1284). Cells were cultured in DMEM (low glucose) supplemented with $10 \%$ (vol/vol) FBS (Sigma-Aldrich) and $100 \mathrm{U} \mathrm{ml}^{-1}$ penicillin-streptomycin (Lonza). B16/LacZ cells $\left(5 \times 10^{5}\right.$ cells) were injected i.v., lungs were collected $19 \mathrm{~d}$ after tumor cell inoculation, lobes were separated, and tumor nodules were quantified on the both sides of each lobule.
Human participants and human cell isolation. Patient samples were obtained from the Children's Hospital and the Institute of Pathology at the Kantonsspital St. Gallen upon receiving informed consent from patients and/or their guardians. The study was approved by the ethics committee of Eastern Switzerland as EKOS 18/113 ('Assessment of target cell tropism of viral vectors in tonsillar explants'), the St. Gallen Lung Biopsy Biobank (EKSG 11/044) and is within the frame of general consent at the Kantonsspital St. Gallen. The first sample was acquired on 13 November 2017, and the last sample was processed on 1 April 2019.

Tonsillar material was collected from healthy male and female children between 3 and 14 years of age that were undergoing routine tonsillectomy. Inform consent was signed by at least one parent or the legal guardian for children from 3 to 13 years of age; for 14-year-old children, the child also signed the informed consent. Individuals presenting the following features were excluded from the study: (1) major craniofacial abnormalities, (2) undergoing immunosuppression or antibiotic treatment, (3) major inflammatory lesions on the tonsils, (4) patients with pre-existing immunocompromising disease. Healthy volunteers (aged 25-35 years) donated blood. Lung tissue obtained from a patient with non-small cell lung cancer (male) undergoing debulking surgery of lung cancer, and adjacent unaffected lung tissue was obtained for the analysis. Skin tissue was obtained from a healthy patient undergoing reduction mammaplasty (female). All adult patients signed informed consent. Tonsillar and lung tissue was mechanically disaggregated, and single-cell suspensions were generated using the gentleMACS technique (Miltenyi Biotec). Skin cells were isolated using the MACS Whole Skin Digestion kit (Miltenyi Biotec). For in vitro culturing of human stromal cells, samples were depleted of leukocytes using anti-CD45 (Miltenyi Biotec) to enrich for stromal cell fractions. Sorted cells were assessed for viability using trypan blue exclusion and cultured in RPMI 1640 with 5\% (vol/vol) FBS (Sigma-Aldrich), $100 \mathrm{U} \mathrm{ml}^{-1}$ penicillin-streptomycin (Lonza) and $16 \mu \mathrm{g} \mathrm{ml}^{-1}$ gentamicin. PBMCs from healthy volunteers were isolated using Ficoll-Paque gradient centrifugation. For in vitro infection, cells were seeded at density of $10^{6}$ cells per well in 48 -well format, rested for $1 \mathrm{~h}$ at $37^{\circ} \mathrm{C}$ and infected with ChAdOx1-GFP or HuAd5-GFP using MOI of 10,100 and 1,000 . The infection was allowed for $3 \mathrm{~h}$, after which the medium was replaced. Cells were kept at $37^{\circ} \mathrm{C}$ overnight and analyzed the next day using flow cytometry.

Short-term culture of primary cells. Tonsillar tissue was extensively washed using PBS with $16 \mu \mathrm{g} \mathrm{ml}^{-1}$ gentamicin. The tissue was cleaned of coagulated blood and cauterized and necrotic areas, and cut into pieces $1 \times 2 \times 1 \mathrm{~mm}$ in size, each containing approximately $10^{6} \mathrm{cell}^{58}$. During all steps, the tissue was maintained in RPMI 1640 with $15 \%$ (vol/vol) FBS (Sigma-Aldrich), 1\% (vol/vol) MEM non-essential amino acids (Gibco), 1\% (vol/vol) MEM sodium pyruvate (Gibco) and $16 \mu \mathrm{g} \mathrm{ml}^{-1}$ gentamicin. Medium-soaked Gelfoam (Pfizer) was used to ensure equally distributed nutrient availability in $1 \times 1-\mathrm{cm}$ pieces. Each well of a six-well plate was filled with $3 \mathrm{ml}$ medium and one Gelfoam piece was deposited per well. Four tonsillar pieces were distributed per Gelfoam piece in each well. Tissue explants were infected by carefully pipetting Ad vectors onto the tissue using $10^{9} \mathrm{IU}$ in 1-2 $\mu$ l per tissue piece (corresponding to an MOI of 1,000 ) or left uninfected. Infection was allowed for $24 \mathrm{~h}$ at $37^{\circ} \mathrm{C}$, after which tissue pieces were collected and briefly washed with PBS-gentamicin before storing overnight in $4 \%$ PFA at $4^{\circ} \mathrm{C}$. The following day, samples were processed for confocal microscopy.

Flow cytometry. Cell characterization was performed by incubating single-cell suspensions in PBS containing 0.5\% FCS and $10 \mathrm{mM}$ EDTA for $25 \mathrm{~min}$ at $4^{\circ} \mathrm{C}$, using the indicated antibodies (Supplementary Table 1). Antigen-specific T cell were detected using PE-conjugated bgal $\mathrm{P}_{96-103}$ and APC-conjugated bgal $\mathrm{A}_{497-504}$ tetramers (NIH Reagents) after an incubation of $20 \mathrm{~min}$ at $37^{\circ} \mathrm{C}$. Ghost Dye Violet 510 and 7AAD (LubioScience) was used to discriminate dead cells in flow cytometric analyses. For peptide-specific cytokine production, $10^{6}$ lung or spleen-derived lymphocytes were restimulated with the bgal ${ }_{96-103}$ peptide (DAPIYTNV) or bgal ${ }_{497-504}$ (ICPMYARV) (NeoSystems) for $2 \mathrm{~h}$ at $37^{\circ} \mathrm{C}$. After this, brefeldin A $\left(5 \mu \mathrm{g} \mathrm{ml}^{-1}\right)$ was added, and cells were cultured for $5 \mathrm{~h}$ at $37^{\circ} \mathrm{C}$. Cells were stimulated with PMA $\left(50 \mathrm{ng} \mathrm{ml}^{-1}\right)$ and ionomycin $\left(500 \mathrm{ng} \mathrm{ml}^{-1}\right.$; both purchased from Sigma-Aldrich) for the positive control or left untreated for the negative control. For intracellular or intranuclear staining, restimulated cells were surface stained and fixed using Cytofix/Cytoperm (BD Biosciences) or the Foxp3/Transcription Factor Staining Buffer Set (eBioscience) for $20 \mathrm{~min}$. Fixed cells were incubated at $4{ }^{\circ} \mathrm{C}$ for $40 \mathrm{~min}$ with monoclonal anti-IFN- $\gamma$ and anti-TNF (eBioscience) diluted in permeabilization buffer ( $2 \% \mathrm{FCS}, 0.5 \%$ saponin, PBS). Alternatively, cells were incubated in permeabilization buffer containing anti-Bcl-2 (BioLegend), anti-Ki67 (eBioscience) or anti-TOM20 (Abcam) at $4^{\circ} \mathrm{C}$ for $30 \mathrm{~min}$. MitoTracker staining was performed according to the manufacturer's instructions (Life Technologies). Samples were analyzed by flow cytometry using a FACSFortessa flow cytometer operating programs FACSDiva and FACSChorus (BD Biosciences). Data were analyzed using FlowJo software (Tree Star).

RNA isolation and quantitative PCR with reverse transcription. T cells were sorted using antibodies to CD8 (FITC), 7AAD (for live-dead staining) and the PE-conjugated bgal ${ }_{96-103}$ tetramer (NIH Reagents), and gating strategies are shown in Extended Data Fig. 2d. FSCs were sorted using antibodies to 
CD45, CD31, EpCAM and Ter119 (PeCy7); CD34 (APC); Sca-1 (APC-Cy7); and 7AAD for live-dead distinction (Supplementary Table 1), and the gating strategy is shown in Extended Data Figs. 5a and 6d. RNA was isolated using the Quick-RNA Miniprep kit (Zymo Research). Contaminating DNA was eliminated by on-column DNase digestion (Zymo Research). cDNA was generated using the QuantiTect Reverse Transcription kit (Qiagen), and quantitative RT-PCR was performed with LightCycler 480 SYBR Green I Master Mix or LightCycler TaqMan Master on a QuantStudio 3 machine (Applied Bioscience) using primers for Il33 (QT00135170), lacZ (Ac03987581_mr and Mr03987581_mr), Uqcrc2 (Mm00445961_ml), Sdha (Mm01352366_ml) and Cox4i1 (Mm01250094_m1). Relative gene expression analysis was performed using the $\Delta \Delta \mathrm{Ct}$ method. Relative expression of samples from immunized and unimmunized lung tissue was calculated using the comparative cycle threshold method based on normalized expression of the housekeeping gene Hprt (QT00166768). To determine absolute gene expression, a negative control, containing reagents only, and serial dilutions of plasmid containing the specific lac $Z$ sequence were included in each run to generate a standard curve. Concentrations of the plasmid dilutions were 280,000 , $28,000,2,800,280$ and 28 copies per reaction. lacZ mRNA concentration in the unknown samples was calculated using data from the standard curve. Final copy numbers were calculated per 1,000 sorted cells.

Immunohistochemistry and confocal microscopy. Murine tissue or human tonsillar tissue pieces were fixed overnight at $4{ }^{\circ} \mathrm{C}$ in freshly prepared $4 \%$ paraformaldehyde (Merck Millipore) under agitation. Tissues were embedded and oriented in $4 \%$ low-melting agarose (Invitrogen) in PBS and serially sectioned with a vibratome (VT-1200, Leica). Sections (30-120 $\mu$ m thick) were collected and blocked in PBS containing 10\% FCS, $1 \mathrm{mg} \mathrm{ml}^{-1}$ anti-Fcy receptor (BD Biosciences) or anti-human Fc receptor (Miltenyi Biotec) and 0.1\% Triton X-100 (Sigma). Tissues were incubated overnight at $4^{\circ} \mathrm{C}$ with the following antibodies: anti-CD31 (Thermo Fisher), anti-CD8, anti-B220 (eBioscience), anti-ACTA2 (Thermo Fisher), anti-EYFP (Takara), anti-IL-33 (R\&D Systems). Human tonsillar tissue explants were characterized using anti-PDPN (eBioscience) and anti-CD45 (BD Biosciences). Unconjugated antibodies were detected with the following secondary antibodies: Alexa Fluor 488-conjugated donkey anti-rabbit IgG, Alexa Fluor 647-conjugated goat anti-rat IgG and Alexa Fluor 594-conjugated goat anti-rat IgG (all purchased from Jackson Immunotools). Nuclei were stained with 4'-6-diamidino-2-phenylindole (DAPI) dihydrochloride (Life Technologies), and sections were mounted on glass microscopy slides using fluorescence mounting medium (Dako). Microscopy of immunofluorescent specimens was performed with an LSM 710 confocal microscope (Carl Zeiss), and microscopy data were recorded and processed with ZEN software (Zeiss). Acquired $z$ stacks were reconstructed and processed for noise removal and 3D-rendering in Imaris version 9 (Bitplane) software.

\section{Quantification of GFP-expressing cells in tonsillar tissue explants. Confocal} microscopy images of human tonsillar tissue explants infected with GFP-expressing Ad vectors were processed using Imaris (Bitplane). DAPI staining was used to identify cell nuclei belonging specifically to single GFP-expressing (infected) cells and to determine the number of infected cells per imaged area ( $\times 40$ objective). The object-placing function in Imaris was used to label and quantify all GFP-expressing cells. $\mathrm{GFP}^{+}$cells were inspected for expression of PDPN or CD45 and marked for automated quantification. The fraction of Ad-infected PDPN- or CD45-expressing cells was calculated as the percentage of all $\mathrm{GFP}^{+}$cells quantified per imaged area.

Cell sorting, library preparation and single-cell RNA-seq analysis. Flow cytometry sorting was used to isolate pulmonary $\mathrm{EYFP}^{+}$cells, while excluding $\mathrm{CD}^{2} 5^{+}, \mathrm{CD} 31^{+}, \mathrm{EPCAM}^{+}$and Ter $119^{+}$cells based on the gating strategy shown in Extended Data Fig. 5a and using the antibodies listed in Supplementary Table 1. Lung-associated, bgal ${ }_{96}$-tetramer-binding $\mathrm{CD} 8^{+} \mathrm{T}$ cells were isolated from the lungs using antibodies to $\mathrm{CD} 8$ (FITC), 7AAD (for live-dead staining) and the bgal $_{96-103}$ tetramer (PE conjugated) (NIH Reagents), and gating strategies are shown in Extended Data Fig. 2d. Sorted cells were run using the 10x Chromium (10x Genomics) system, and cDNA libraries were generated according to the manufacturer's recommendations (Chromium Single Cell 3' Reagent Kit (version 2 chemistry and version 3 chemistry)). Sequencing of libraries was performed using the NextSeq 500 or NovaSeq 6000 Illumina sequencing system at the Functional Genomic Center Zürich. Initial processing and gene expression estimation were performed using Cell Ranger (version 3.0.2) with the Ensembl GRCm38.94 release as a reference to build index files for alignments. This preprocessing resulted in UMI counts for a total of 17,415 FSCs and 10,075 T cells. The two datasets were analyzed separately, and samples were pooled from multiple replicates from at least two independent experiments (FSCs, naive $C c l 19-c r e ; R 26 R^{\mathrm{EXFP}}$ controls, two biological replicates; immunized $C c l 19-c r e ; R 26 R^{\mathrm{EYFP}}$ mice, two biological replicates; $\mathrm{T}$ cells, lung-associated $\mathrm{CD}^{+} \mathrm{T}$ cells isolated from Ad-LacZ/FlexON-immunized Ccl19-cre mice, two biological replicates; lung-associated $\mathrm{CD} 8^{+} \mathrm{T}$ cells isolated

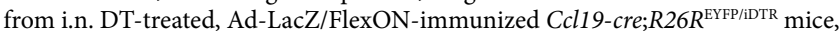
two biological replicates) with batches spanning multiple conditions and all conditions represented by multiple batches. The scater R/Bioconductor package (version 1.14.1) was used for subsequent quality control ${ }^{59}$, which was completed in $\mathrm{R}$ version 3.6.1 and included the removal of cells with particularly high or low numbers of detected genes or UMI counts (more than two median absolute deviations from the median across all cells) or a large fraction of mitochondrial genes (more than two median absolute deviations above the median across all cells) as described in ref. ${ }^{53}$. Moreover, cycling cells were excluded from downstream analysis based on the expression of genes Top2a, Mki67, Cenpf or Pclaf. Contaminating hematopoietic cells, endothelial cells and epithelial cells were excluded based on the expression of Pecam1, Krt18, Lyve1, Ptprc, Cd79a, $C d 3 e, C l d n 5$ or $C d 53$, and only cells expressing mRNA for EYFP were kept for downstream analysis. After quality control and removal of contaminants, 5,356 FSCs $\left(2,465\right.$ cells from naive Ccl19-cre; $R 26 R^{\mathrm{EYFP}}$ controls; 2,891 cells from immunized Ccl19-cre;R26R ${ }^{\mathrm{EYFP}}$ samples) and 8,393 T cells (7,211 cells from PBS-treated mice; 1,182 cells from DT-treated mice) were retained for further processing using the Seurat package (version 3.1.1) ${ }^{60}$. Because data were collected and processed in two batches, canonical correlation analysis was used to integrate data from different batches by running the following steps as implemented in Seurat: normalization of UMI counts, regression to remove the influence of UMI counts per cell and detection of highly variable genes per batch. Data integration was performed using FindIntegrationAnchors and IntegrateData functions based on the first six correlation components for FSCs and the first nine correlation components for $\mathrm{T}$ cells.

Extended bioinformatic analyses. Dimensional reduction and graph-based clustering were performed on integrated data, and FSC clusters were characterized on the basis of canonical mesenchymal cell markers and unbiased marker genes. Marker genes for each FSC cluster as well as differentially expressed genes between conditions were inferred by the Wilcoxon test as implemented in the FindMarker function of Seurat ${ }^{60}$, and top significant genes were summarized in gene signatures based on their reported function. To investigate functional differences between $\mathrm{T}$ cells from DT- and PBS-treated mice, differentially expressed genes were tested for enrichment in gene ontologies using the 'enrichGO' function from the clusterProfiler R/Bioconductor package (version 3.16.0) ${ }^{61}$. Top significant ontologies $(q$ value $<0.05)$ were visualized by running the 'cnetplot' function. To analyze the dynamics of fibroblast differentiation, we ran velocyto.py (version $0.17 .17)^{40}$ as a tool to predict future cell states based on the ratio of unspliced to spliced reads. Briefly, loom files were generated by running 'velocyto run $10 \mathrm{x}$ ' on Cell Ranger output and used to infer spliced and unspliced assay data. RNA velocity was estimated using the 'RunVelocity' function from the SeuratWrappers $\mathrm{R}$ package (version 0.1.0). Furthermore, cluster similarities were calculated as Pearson correlation between clusters based on the average expression across all genes.

Statistical analyses. All statistical analyses were performed with Prism 8.4.3 (GraphPad). Unless specified otherwise, graphs depict mean \pm s.e.m. Differences between two groups were evaluated using unpaired two-tailed Student's $t$-tests or one- or two-way ANOVA. Statistical methods used are indicated in the figure legends. Exact $P$ values are present in the source data. Results were considered statistically significant when ${ }^{*} P<0.05,{ }^{* *} P<0.01,{ }^{* * *} P<0.001$ and ${ }^{* * * *} P<0.0001$.

Reporting Summary. Further information on research design is available in the Nature Research Reporting Summary linked to this article.

\section{Data availability}

scRNA-seq data are available in the ArrayExpress database (accession numbers E-MTAB-9558 and E-MTAB-9580). Ensembl GRCm38.94 was used as a reference to build index files for alignments in scRNA-seq analysis. Further information and requests for resources should be directed to and will be fulfilled by the lead contacts B.L. (burkhard.ludewig@kssg.ch) and P. Klenerman (paul.klenerman@ medawar.ox.ac.uk). Source data are provided with this paper.

\section{References}

57. Alharbi, N. K. et al. ChAdOx1 and MVA based vaccine candidates against MERS-CoV elicit neutralising antibodies and cellular immune responses in mice. Vaccine 35, 3780-3788 (2017).

58. Grivel, J. C. \& Margolis, L. Use of human tissue explants to study human infectious agents. Nat. Protoc. 4, 256-269 (2009).

59. McCarthy, D. J., Campbell, K. R., Lun, A. T. \& Wills, Q. F. Scater: pre-processing, quality control, normalization and visualization of single-cell RNA-seq data in R. Bioinformatics 33, 1179-1186 (2017).

60. Stuart, T. et al. Comprehensive integration of single-cell data. Cell 177, 1888-1902 (2019).

61. Yu, G., Wang, L. G., Han, Y. \& He, Q. Y. clusterProfiler: an R package for comparing biological themes among gene clusters. OMICS 16, 284-287 (2012).

\section{Acknowledgements}

We thank S. Caviezel-Firner and C. Engetschwiler for excellent technical support. This study received financial support from the Swiss National Science Foundation (grants 
166500 and 159188 to B.L.), Swiss Cancer Research (KFS-4162-02-2017-R to P. Krebs), the Wellcome Trust (109965MA to P. Klenerman) and research fellowship grants from the British Infection Association (to J.M.C.) and the Wellcome Trust (099897/Z/12/A to J.M.C.).

\section{Author contributions}

B.L., P. Klenerman and J.C. designed the study, discussed data and wrote the paper. J.C. J.M.C., S.S.R., L.O., A.D.M., H.-W.C. and D.E. conducted experiments and discussed data. M.L. performed bioinformatic analyses and discussed data. N.M.P. discussed data and provided reagents. P. Krebs and A.O. discussed data and provided reagents. L.F. and E.S. discussed data.

\section{Competing interests}

L.F. is a cofounder and shareholder of Hookipa Pharma. B.L., L.O. and H.-W.C. are cofounders and shareholders of Stromal Therapeutics. S.S.R. and H.-W.C. are part-time employees of Stromal Therapeutics. The remaining authors declare no competing interests.

\section{Additional information}

Extended data is available for this paper at https://doi.org/10.1038/s41590-021-00969-3.

Supplementary information The online version contains supplementary material available at https://doi.org/10.1038/s41590-021-00969-3.

Correspondence and requests for materials should be addressed to P.K. or B.L

Peer review information Nature Immunology thanks Stephen Jameson and the other, anonymous, reviewer(s) for their contribution to the peer review of this work. Peer reviewer reports are available. L. A. Dempsey was the primary editor on this article and managed its editorial process and peer review in collaboration with the rest of the editorial team.

Reprints and permissions information is available at www.nature.com/reprints. 
a

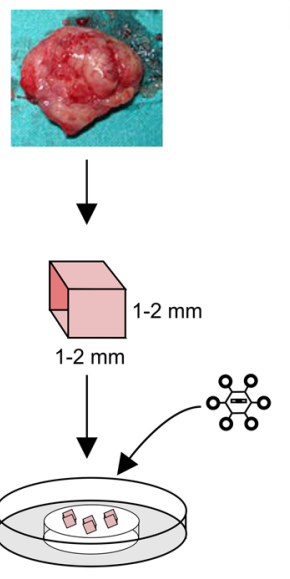

b

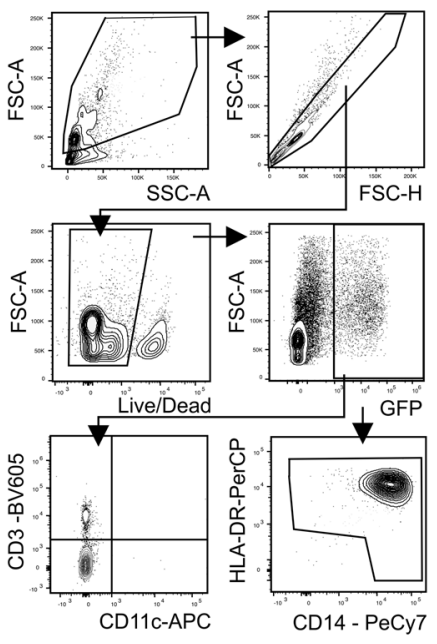

C

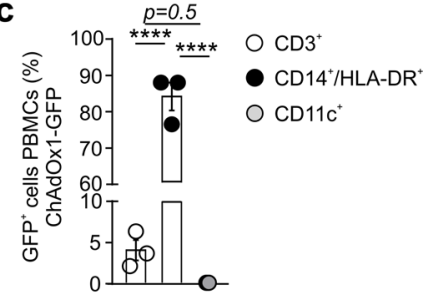

d

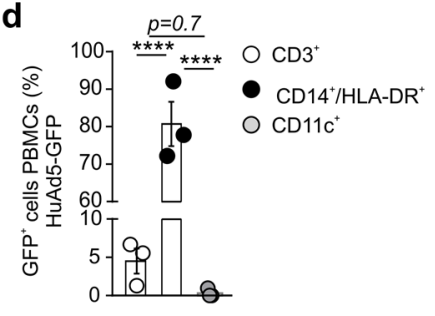

e
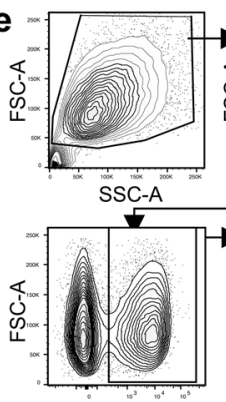

GFP

h Cultured skin stromal cells
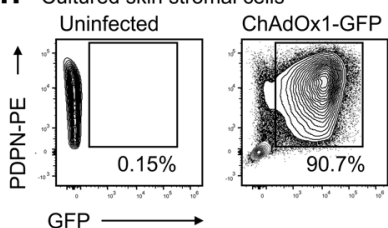

GFP

K Cultured lung stromal cells

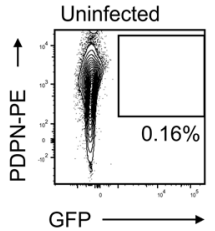

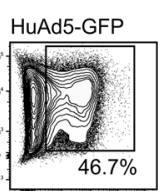

$46.7 \%$

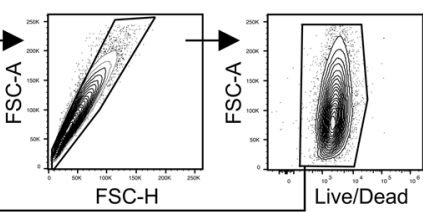

f

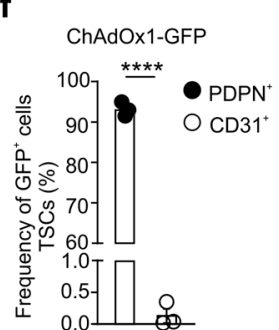

g

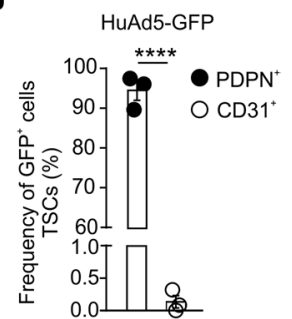

i

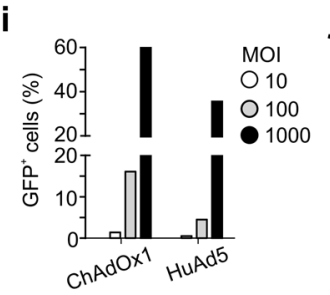

I

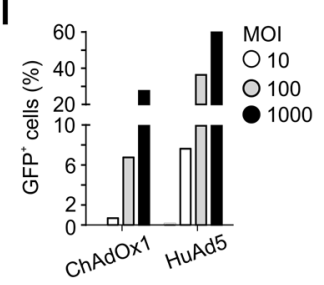

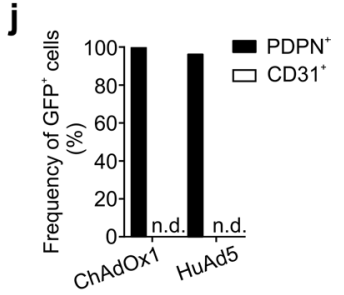

m

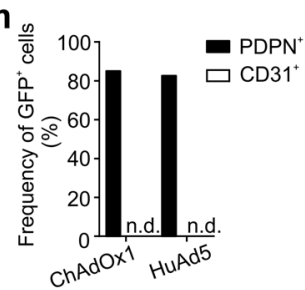

Extended Data Fig. 1 | Adenoviral vectors ChAdOx1 and HuAd5 transduce human fibroblasts. a, Preparation of sliced tissue cultures from human palatine tonsils following infection with adenoviral vectors. b, Flow cytometry-based gating strategy for PBMCs analysis. $\mathbf{c}$ and $\mathbf{d}$, Frequency of CD3+ T cells, CD14+ HLA-DR ${ }^{+}$monocytes or CD11c dendritic cells (DCs) within GFP+ cells following infection with (c) ChAdOx1-GFP or (d) HuAd5-GFP. e, Flow cytometry-based gating strategy for cultured tonsillar tissue stromal cell analysis. $\mathbf{f}$ and $\mathbf{g}$, Frequencies of PDPN ${ }^{+}$and $\mathrm{CD} 1^{+}$cells within GFP+ cultured tonsillar tissue stromal cells (TSC) following infection with (f) ChAdOx1-GFP or ( $\mathbf{g}$ ) HuAd5-GFP. $\mathbf{h}$ to $\mathbf{m}$, Infection of cultured (h to $\mathbf{j}$ ) skin- or ( $\mathbf{k}$ to $\mathbf{m}$ ) lung-derived stromal cells with ChAdOx1-GFP or HuAd5-GFP with representative FACS plots. (i and I) Frequency of GFP+ cells after infection with indicated adenoviral vectors at different multiplicity of infection (MOI). ( $\mathbf{j}$ and $\mathbf{m}$ ) Frequency of PDPN ${ }^{+}$or $\mathrm{CD}_{31}{ }^{+}$cells within $\mathrm{GFP}^{+}$cells after infection with indicated adenoviral vectors. Pooled data from $n=3$ PBMC samples $[(c)$ and $(d)]$ and $n=3$ TSC samples $[(f)$ and $(g)]$. Data from one experiment with single skin $[(\mathrm{h}-\mathrm{j})]$ or lung tissue donor $[(\mathrm{k}-\mathrm{m})]$. Statistical analysis was performed using one-way ANOVA with Tukey's multiple comparison test $[(\mathrm{c})$ and $(\mathrm{d})]$ and unpaired two-tailored Student's $t$ test $[(\mathrm{f})$ and $(\mathrm{g})]$, with ${ }^{\star} \mathrm{P}<0.05$; ${ }^{\star \star} \mathrm{P}<0.01$; ${ }^{\star \star \star} \mathrm{P}<0.001$; ${ }^{\star \star \star \star} \mathrm{P}<0.0001$. Exact $\mathrm{P}$ values are provided in the Source Data. 
a

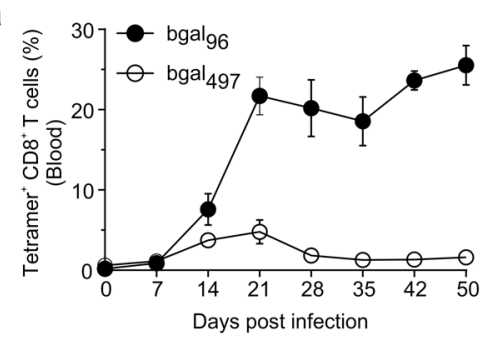

b

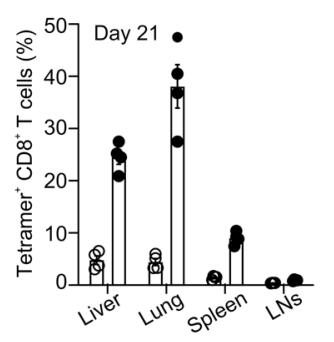

C

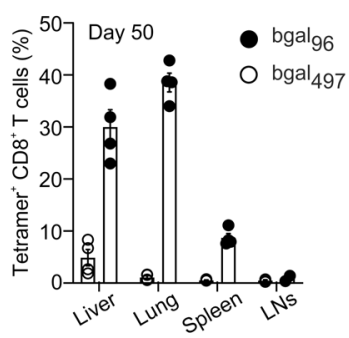

d

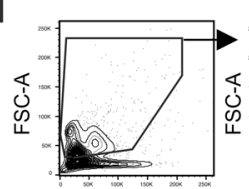

SSC-A

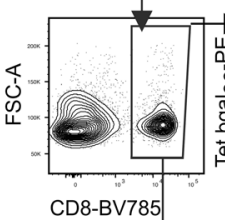

CD8-BV785

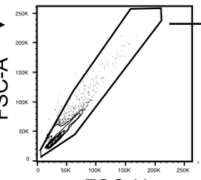

FSC-H

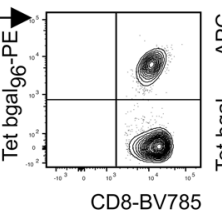

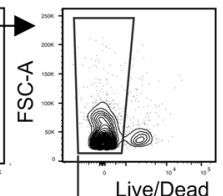

Live/Dead

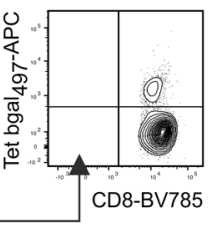

e

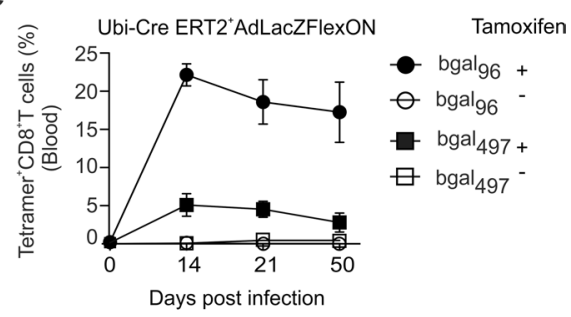

f

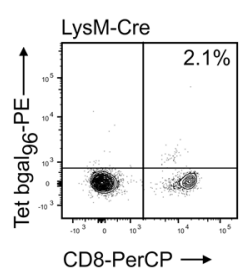

g

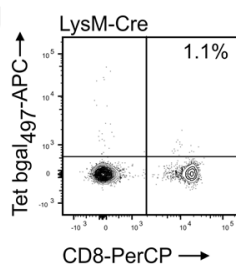

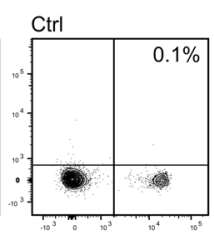

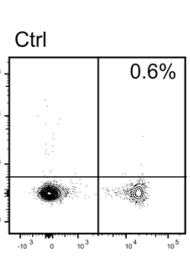

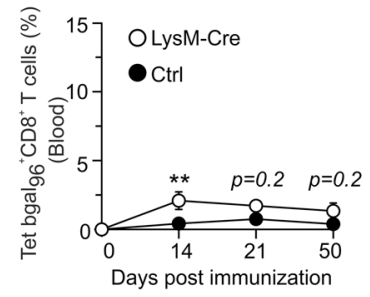

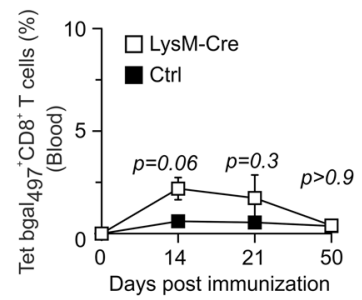

Extended Data Fig. 2 | Induction of inflationary memory CD8 ${ }^{+}$T cells following Ad5-LacZ immunization. a to c, B6 mice were immunized i.v. with Ad-LacZ. (a) Kinetics of the frequency of bgal ${ }_{96}$ or bgal $_{497}$ tetramer $^{+} \mathrm{CD} 8^{+} \mathrm{T}$ cells in blood. Frequency of bgal ${ }_{96}$ or bgal $_{497}$ tetramer $^{+} \mathrm{CD} 8^{+} \mathrm{T}$ cells in indicated organs on (b) day 21 or (c) day 50. d, Flow cytometry-based gating strategy for tetramer ${ }^{+} \mathrm{CD} 88^{+} \mathrm{T}$ cell analysis. e, Ubi-Cre ERT2 mice were immunized i.v. with Ad-LacZ/FlexON. Frequency of bgal ${ }_{96}$ or bgal ${ }_{497}$ tetramer ${ }^{+} \mathrm{CD} 8^{+} \mathrm{T}$ cells in blood after application of Tamoxifen. (f to $\mathbf{g}$ ) LysM-Cre mice were immunized i.v. with Ad-LacZ/FlexON. f, Kinetics of bgal ${ }_{96}$ tetramer ${ }^{+} \mathrm{CD} 8^{+} \mathrm{T}$ cell response with representative FACS plots. $\mathbf{g}$, Kinetics of non-inflationary bgal ${ }_{497}$ tetramer ${ }^{+} \mathrm{CD} 8^{+} \mathrm{T}$ cell response with representative FACS plots. Cre-negative mice were used as controls (Ctrl). Pooled data from 2 independent experiments with $n=4-8$ mice $[(a)] ; n=4$ mice per group $[(b)$ and (c) ], representative of two experiments with $n=2$ per group $[(e)]$, $\mathrm{n}=8$ ( LysM-Cre $)$ and $\mathrm{n}=8(\mathrm{Ctrl})[(\mathrm{f})$ and $(\mathrm{g})]$. Values indicate mean \pm s.e.m. for each time point. Statistical analysis was performed using two-way analysis of variance (ANOVA) with Bonferroni multiple comparison test $[(f)$ to $(g)]$ with ${ }^{\star} P<0.05 ;{ }^{\star \star} P<0.01 ;{ }^{* \star \star} P<0.001$; ${ }^{\star \star \star \star} P<0.0001$. Exact $P$ values are provided in the Source Data. 
a

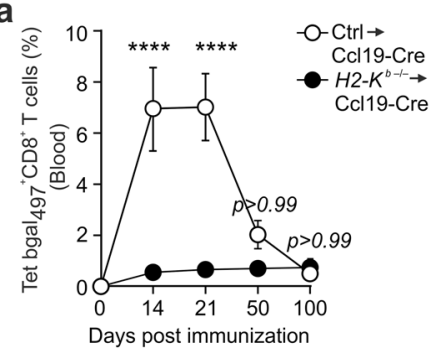

b

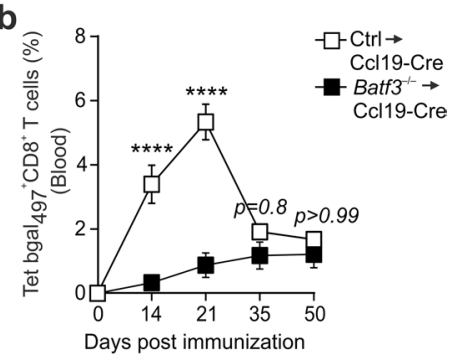

Extended Data Fig. 3 | Cross-talk between Ccl19-Cre ${ }^{+}$cells and professional antigen presenting cells during the induction of LacZ-specific T cell responses. The indicated bone marrow chimeric mice were generated and vaccinated i.v. with Ad-LacZ/FlexON. (a and $\mathbf{b})$ The frequency of bgal ${ }_{497}$ tetramer ${ }^{+} \mathrm{CD} 8{ }^{+} \mathrm{T}$ cells was monitored in blood. Values indicate mean \pm s.e.m. for each time point. Pooled data from 2 independent experiments with $\mathrm{n}=10$ (Ctrl to Ccl19-Cre) to 8 ( $\mathrm{H} 2-\mathrm{K}^{\mathrm{b}-/-}$ to Ccl19-Cre) mice $[(\mathrm{a})]$ and $\mathrm{n}=5$ (Ctrl to Ccl19-Cre) and 5 (Batf3-/- to Ccl19-Cre) mice [(b)]. Statistical analysis was performed using two-way analysis of variance (ANOVA) with Bonferroni multiple comparison test $[(a)$ and $(b)]{ }^{\star} P<0.05$; ${ }^{\star \star} P<0.01$; ${ }^{\star \star \star} P<0.001$; ${ }^{\star \star \star \star} P<0.0001$. Exact $P$ values are provided in the Source Data. 
a

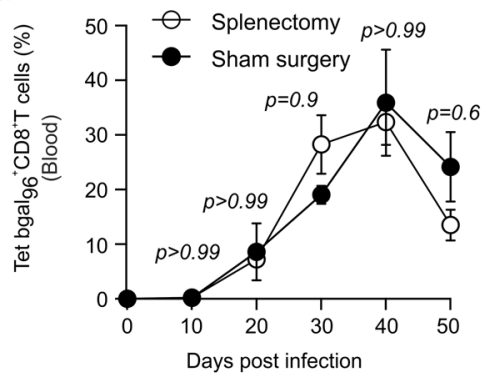

b

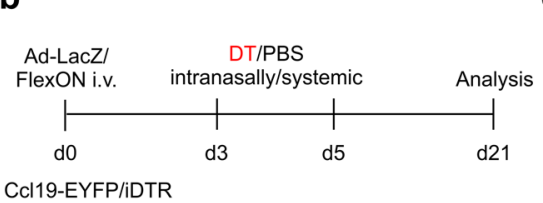

c

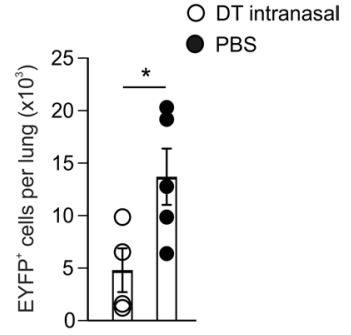

d
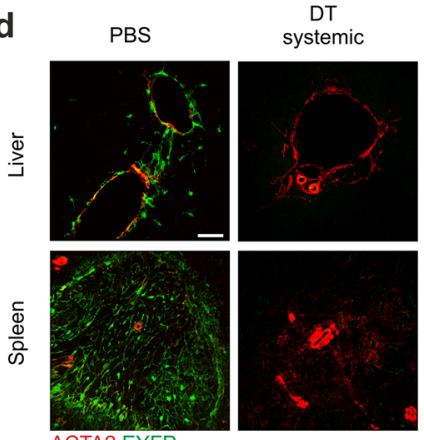

ACTA2 EYFP
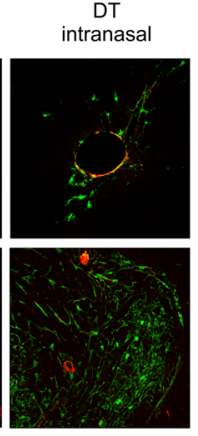

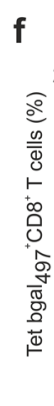

g

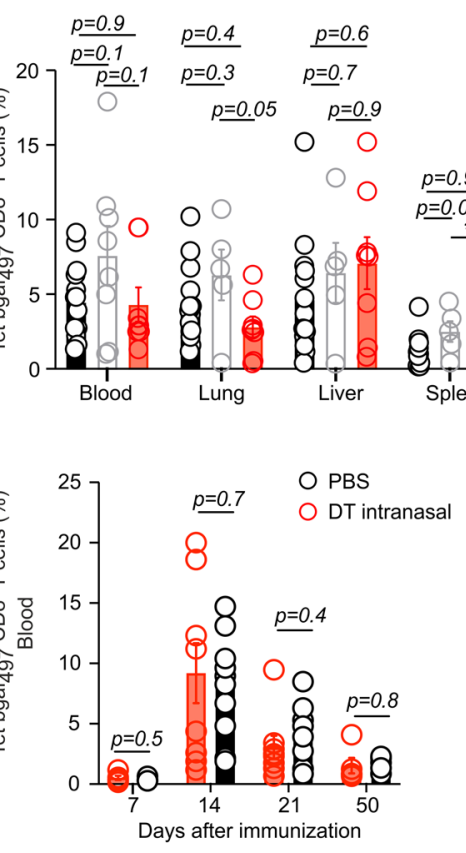

O PBS

DT systemic

O DT intranasal e

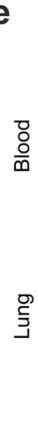

$\stackrel{\bar{\Xi}}{3}$
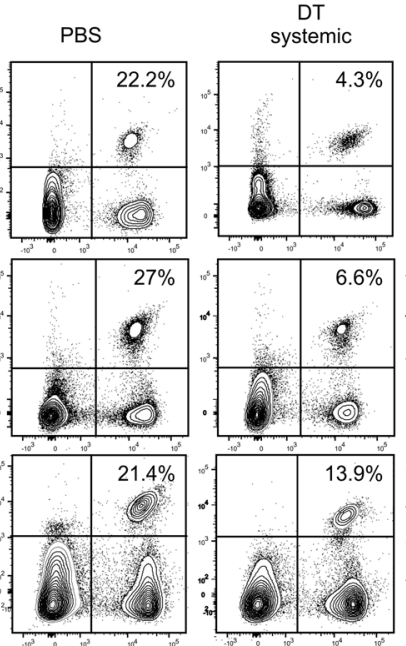

के
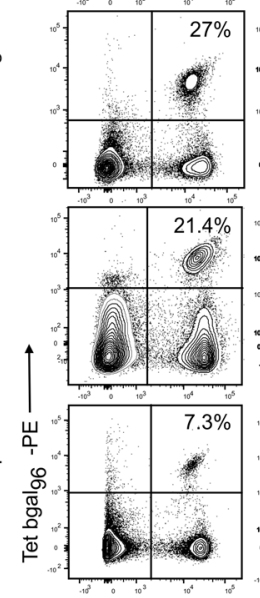

CD8-BV785
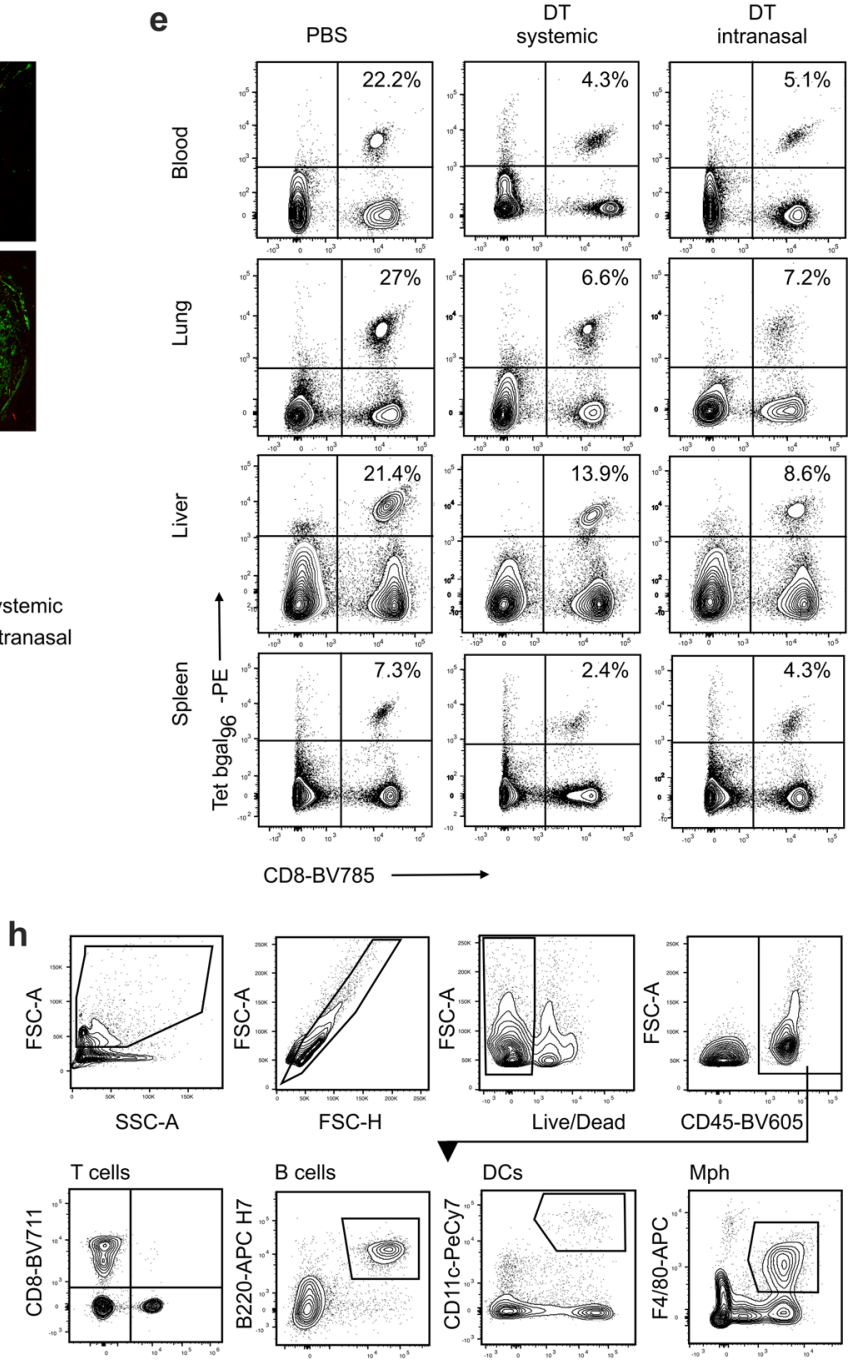

I-ËII-A-BV421

CD11b-FITC

Extended Data Fig. 4 | 4. Location of Ccl19-Cre ${ }^{+}$cells that contribute to induction and maintenance of memory inflating CD8 ${ }^{+}$T cells. a, Ccl19-EYFP mice were splenectomized and immunized i.v. with Ad-LacZ/FlexON. The frequency of tetramer ${ }^{+} \mathrm{CD} 8^{+} \mathrm{T}$ cells was monitored in blood or the indicated organs. $\mathbf{b}$ to $\mathbf{g}$, Ccl19-EYFP/iDTR mice were vaccinated i.v. with Ad-LacZ/FlexON and DT or PBS were given intranasal (in) or intraperitoneal (sys) on day 3 and 5. (c) Enumeration of EYFP+ cells in the lungs of day 7 Ad-LacZ/FlexON-vaccinated mice, following i.n. DT or PBS application. (d) Representative confocal microscopy image of EYFP+ FSCs in the liver and spleen after systemic or intranasal DT injection. Scale bar equals $30 \mu \mathrm{m}$. (e) Representative FACS plots of bgal ${ }_{96}$ tetramer ${ }^{+} \mathrm{CD} 8+\mathrm{T}$ cells in blood, lung, liver and spleen on day 21 . (f) Frequency of bgal ${ }_{497}$ tetramer $^{+} \mathrm{CD} 8^{+} \mathrm{T}$ cells in the indicated organs. (g) Frequency of bgal ${ }_{497}$ tetramer ${ }^{+} \mathrm{CD} 8^{+} \mathrm{T}$ cells in the blood at the indicated time points. $\mathbf{h}$, Flow cytometry-based gating strategy for lung hematopoietic cells analysis used in Fig.4e. Dots represent individual mice. Bar graphs indicate mean \pm s.e.m. Data from one [(a)] or pooled data from 2 independent experiments [(b) to (g)] with $n=2$ mice per group $[(\mathrm{a})] ; n=4$ (DT intranasal) and $n=5$ (PBS intranasal) $[(\mathrm{c})$ ); representative data from $n=3$ (DT systemic), $n=7$ (DT intranasal) and $n=5$ (PBS control) [(d)]; and $n=5$ (8 blood) (DT systemic), $n=9$ (DT intranasal) and $n=14$ (PBS control) $[(f)], n=5$ (days 7 and 50), 10 mice (day 14) and 8 (day 21) [(g)]. Statistical analysis was performed using two-way analysis of variance (ANOVA) with Bonferroni multiple comparison test [(a)], unpaired two-tailored Student's t test [(c) and $(\mathrm{g})]$ or one-way ANOVA with Tukey's multiple comparison test $[(\mathrm{f})]$ with ${ }^{\star} \mathrm{P}<0.05 ;{ }^{\star \star} \mathrm{P}<0.01$; ${ }^{\star \star \star} \mathrm{P}<0.001$; ${ }^{\star \star \star \star} \mathrm{P}<0.0001$. Exact $\mathrm{P}$ values are provided in the Source Data. 
a

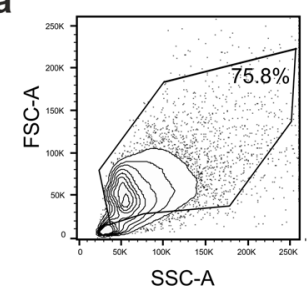

b

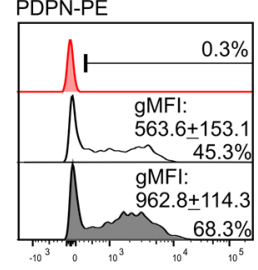

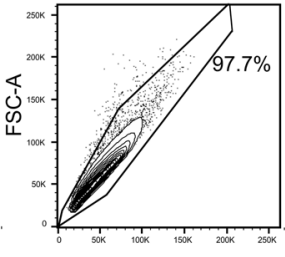

FSC-H

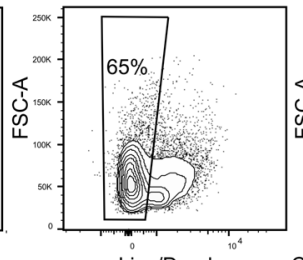

Live/Dead

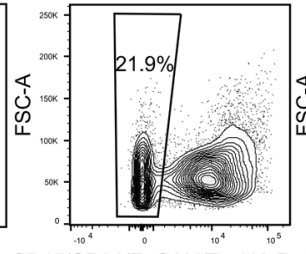

CD45/CD31/EpCAM/Ter119-PeCy7

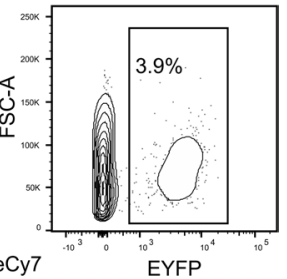

d
O Isotype control

O Naive

- Ad-LacZ/FlexON

day 21

e

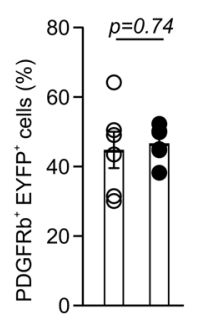

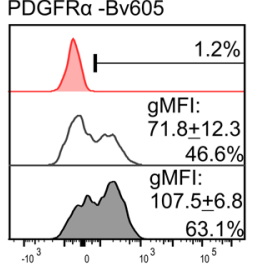

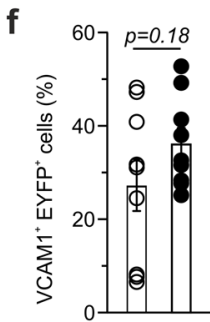

VCAM1 - AlexaFluor 647

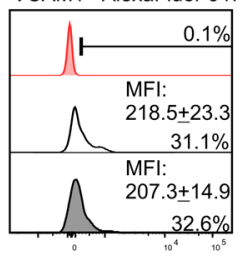

Extended Data Fig. 5 | Phenotypic changes in Ccl19-Cre+ cells after Ad-LacZ/FlexON immunization. a to f, Ccl19-EYFP mice were immunized i.v. with Ad-LacZ/FlexON. On day 21 pulmonary FSCs were analyzed. (a) Gating for analysis of EYFP+ cells. (b to f) Representative FACS histograms with gMFI/MFI and frequencies of (b) $\mathrm{PDPN}^{+}$, (c) ICAM1 ${ }^{+}$, (d) $\mathrm{PDGFRa}^{+}$, (e) $\mathrm{PDGFRb}^{+}$or (f) $\mathrm{VCAM1}^{+}$cells within EYFP+ cells. Dots represent individual mice and bar graphs indicate mean \pm s.e.m. Pooled data from 2 independent experiments with $n=5$ mice per group $[(e)]$ and $n=9$ mice per group [(f)]. Statistical analysis was performed using unpaired two-tailored Student's t test $[(\mathrm{e})$ and $(\mathrm{f})]$ with ${ }^{\star} \mathrm{P}<0.05 ;{ }^{\star \star} \mathrm{P}<0.01$; ${ }^{\star \star \star} \mathrm{P}<0.001$; ${ }^{\star \star \star \star} \mathrm{P}<0.0001$. Exact $\mathrm{P}$ values are provided in the Source Data. 
a
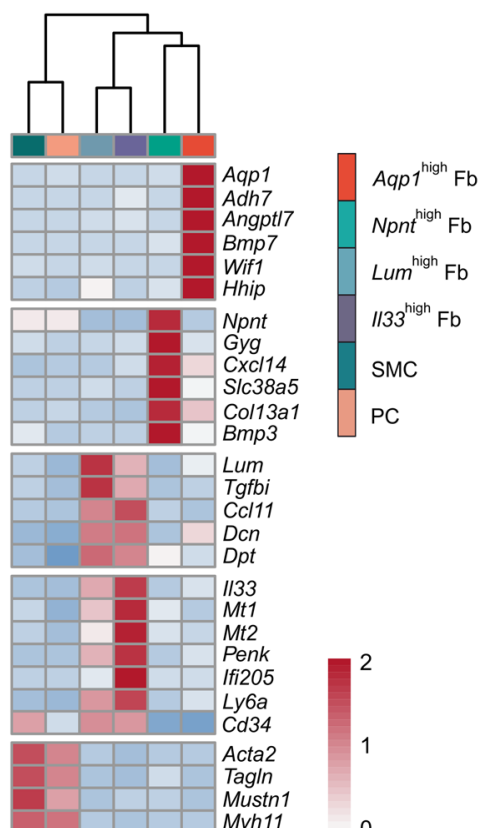

Myh11
Actg2
Des

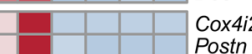

\begin{tabular}{ll}
\hline & Posth \\
Higd1b
\end{tabular}

$\begin{array}{ll}- & \text { Notch3 } \\ \text { Ndufa4l2 } & -2\end{array}$

c

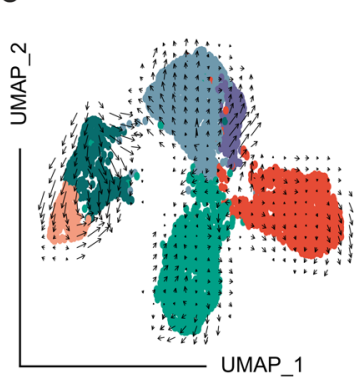

UMAP_1

g

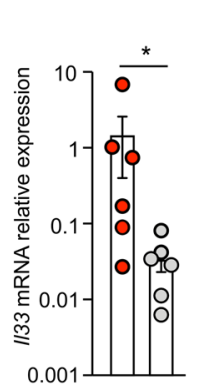

h

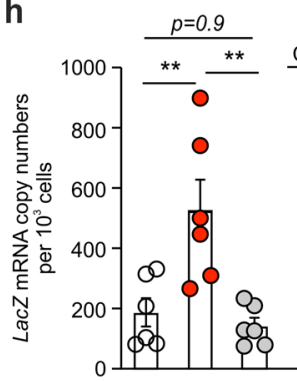

$$
\begin{array}{r}
\text { Aqp }{ }^{\text {nigh }} \mathrm{Fb} \\
\mathrm{Nonht}^{\text {nigh }} \mathrm{Fb} \\
\mathrm{Lum}^{\text {nigh }} \mathrm{Fb} \\
\| 133^{\text {nigh }} \mathrm{Fb} \\
\mathrm{SMC} \\
\mathrm{PC}
\end{array}
$$

b

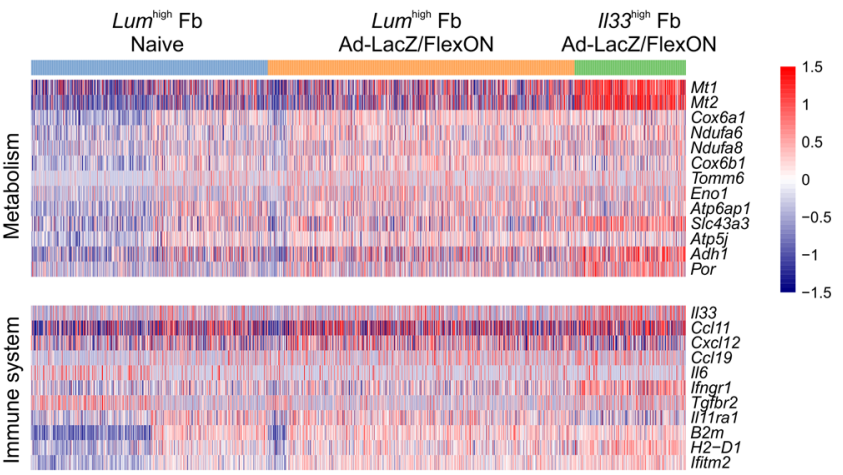

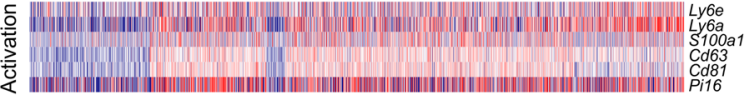
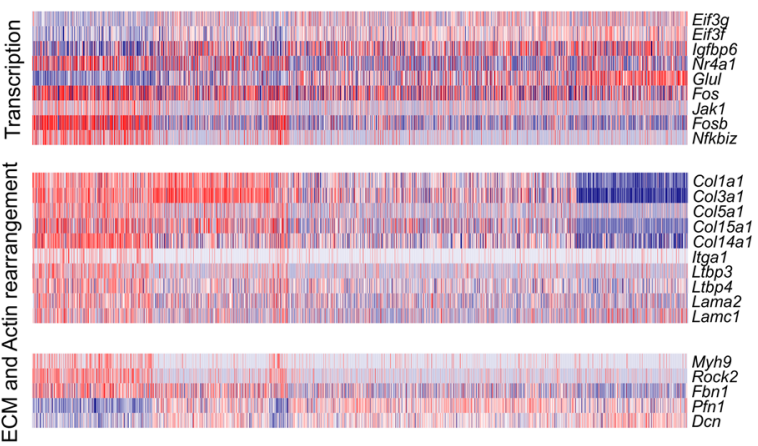

d

Naive
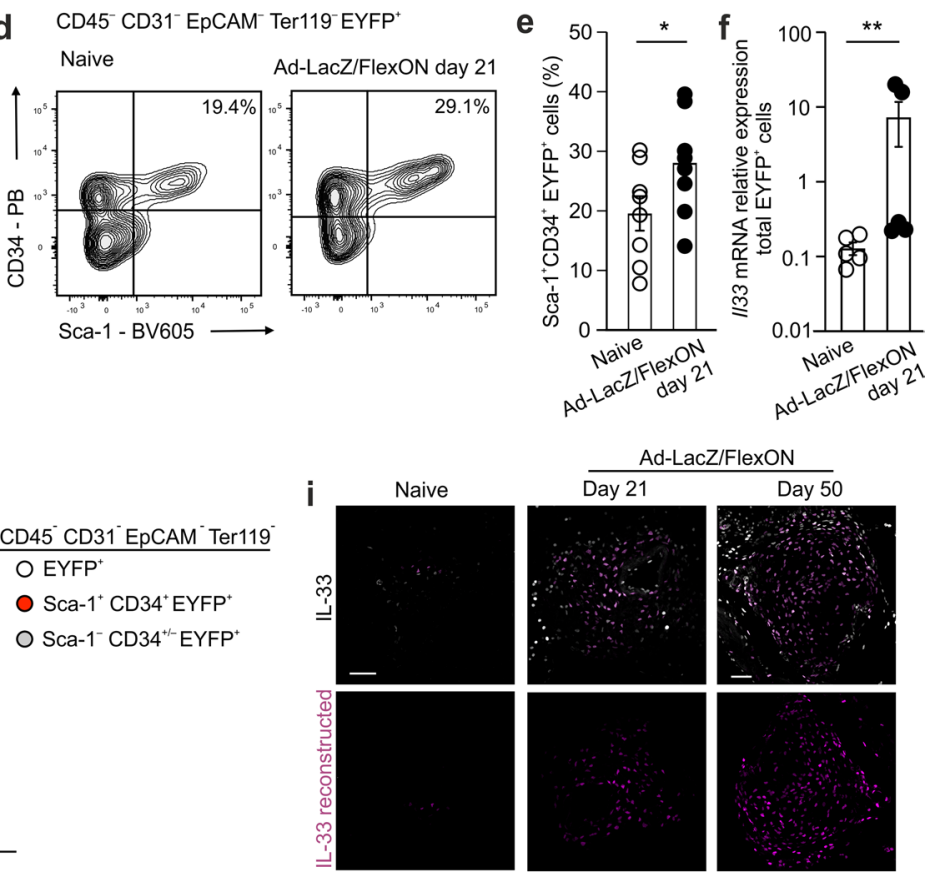

CD45 CD31 EpCAM" Ter $119^{\circ}$ $\mathrm{O} \mathrm{EYFP}^{+}$

O Sca- $1^{+}$CD $34^{+}$EYFP $^{+}$

O Sca- $1^{-}$CD $34^{+/-}$EYFP
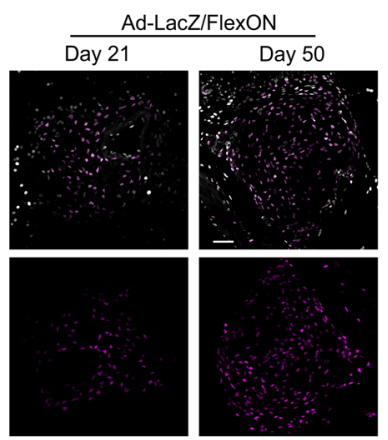

Extended Data Fig. 6 | See next page for caption. 
Extended Data Fig. 6 | Ad-LacZ/FlexON activates pulmonary Ccl19-expressing immune-stimulatory FSCs. a, Averaged expression of cluster specific marker genes for pulmonary FSC clusters in merged naive and Ad-LacZ/FlexON-immunized Ccl19-EYFP mice. b, Heatmaps displaying indicated genes across all Lum ${ }^{\text {high }}$ and II33 high FSCs in EYFP+ FSCs from Ad-LacZ/FlexON-immunized or naive mice. c, Prediction of differentiation dynamics based on RNA velocity analysis of EYFP+ FSCs constructed based on recent changes in the transcriptional rate of a gene to predict the future state of individual cells. d, Representative flow cytometric analysis of Sca $-1^{+} \mathrm{CD} 34^{+}$cell populations within EYFP+ cells. e, Frequency Sca- $1^{+} \mathrm{CD} 34^{+}$cell within EYFP ${ }^{+}$cells. f, Quantitative real-time PCR analysis of $/ 133$ expression in pulmonary EYFP+ FSCs. $\mathbf{g}$, Quantitative real-time PCR analysis of $/ 133$ expression in EYFP+ pulmonary FSC populations. h, Quantitative real-time PCR analysis of LacZ mRNA copy numbers per 1,000 cells in the indicated EYFP+ pulmonary FSC populations. i, Reconstruction of IL-33 staining in lung EYFP+ cells of Ccl19-EYFP mice at indicated time points after Ad-LacZ/FlexON immunization, based on images shown in Fig. 5j. Scale bar equals $30 \mu \mathrm{m}$. Dots represent individual mice and bar graphs indicate mean \pm s.e.m. Pooled data from two independent experiments with $n=8$ naive and 9 AdLacZFlexON immunized $[(\mathrm{e})] ; n=5[(\mathrm{f})], \mathrm{n}=6[(\mathrm{~h})]$ mice; one experiment $\mathrm{n}=6$ mice $[(\mathrm{g})]$. Representative data from $n=4$ mice per group (i). Statistical analysis was performed using unpaired two-tailored Student's $t$ test [(e)], Mann Whitney $[(f),(g)]$, one-way ANOVA with Turkey's multiple comparison test $[(h)]$ with ${ }^{\star} P<0.05 ;{ }^{* \star} P<0.01 ;{ }^{\star \star \star} P<0.001$; ${ }^{\star \star \star \star} P<0.0001$. Exact $P$ values are provided in the Source Data. 
a

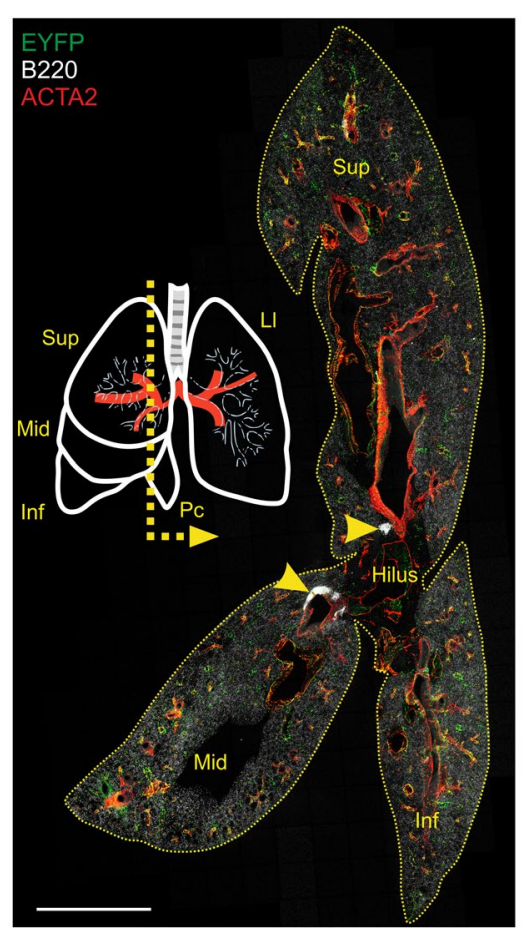

b

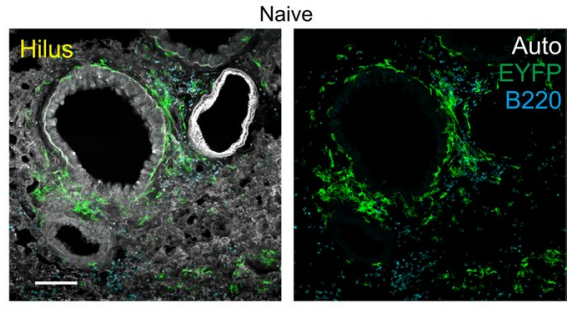

Ad-LacZ/FlexON (Day 21)

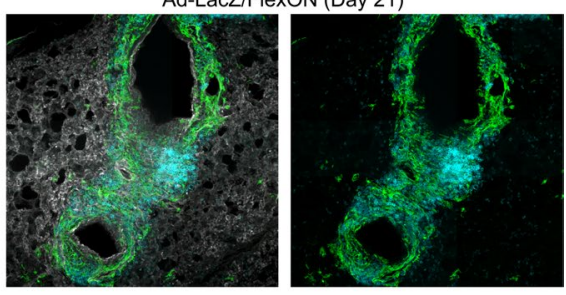

Ad-LacZ/FlexON (Day 50)

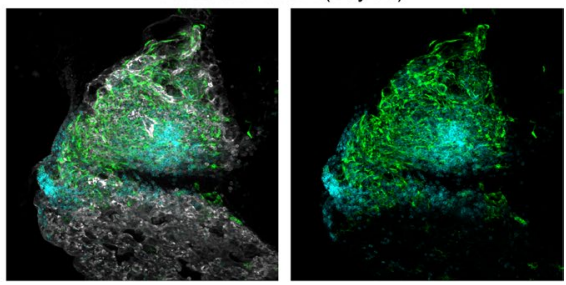

Extended Data Fig. 7 | Bronchus-associated lymphoid tissues in the lungs of Ad-LacZ/FlexON immunized Ccl19-EYFP mice. a, Overview image of three lung lobes (Sup, superior; Inf, inferior; Mid, middle) of the right lung. Dashed line in the schematic indicates approximate sagittal plane of sectioning. Arrowheads indicate lymphoid cell aggregates in the hilus region. Scale bar equals $2 \mathrm{~mm}$. b, Images show hilus regions in lungs of Ccl19-EYFP mice. Scale bar equals $100 \mu \mathrm{m}$. Representative of 2 independent experiments, $n=4$ mice per condition. 
a

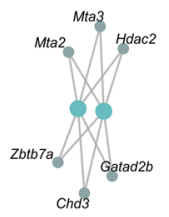

PBS

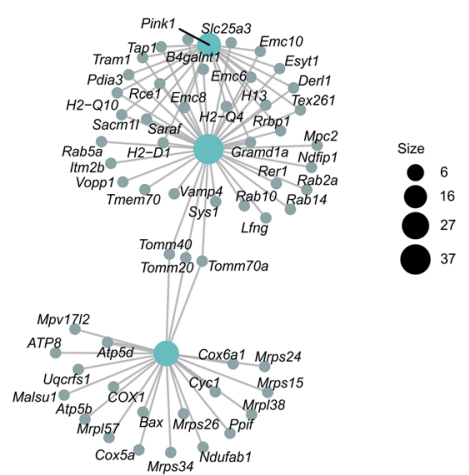

DT

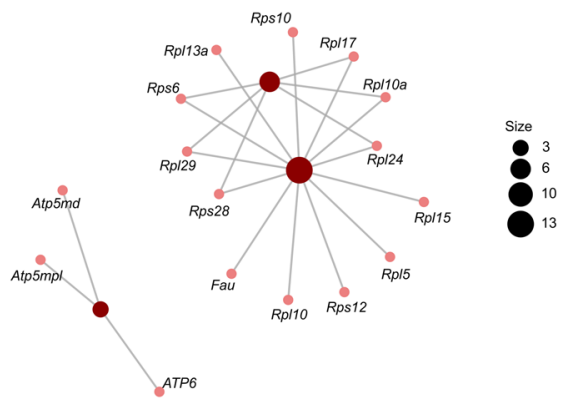

b
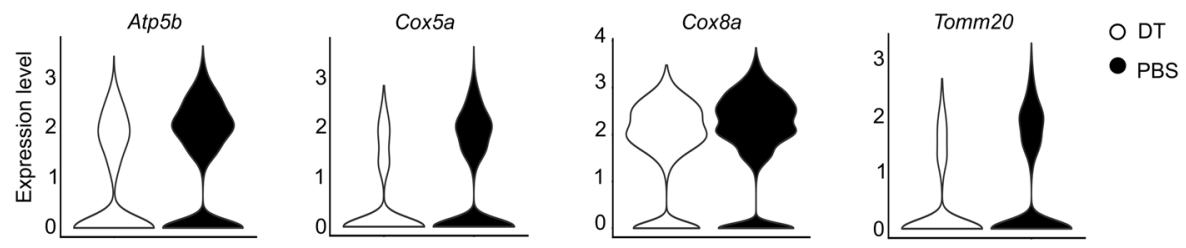

C
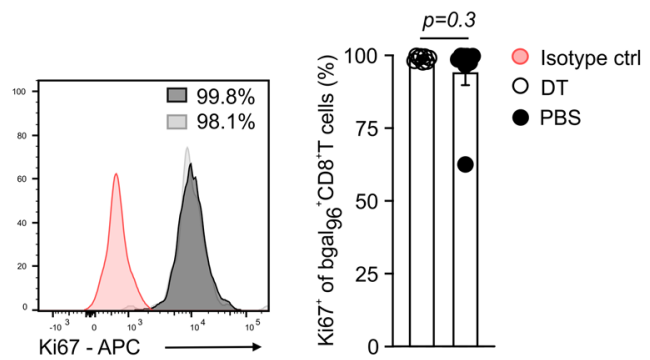

d

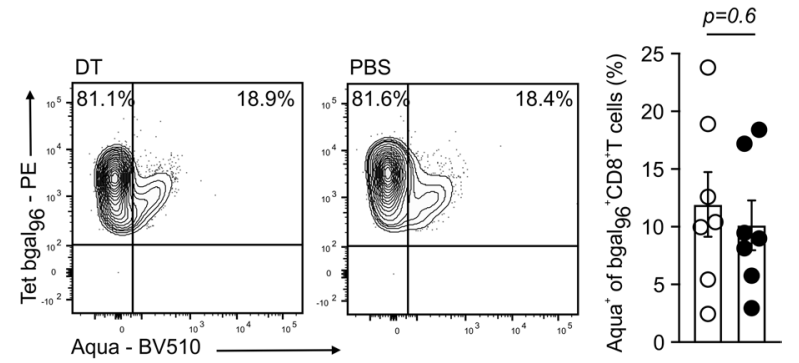

e

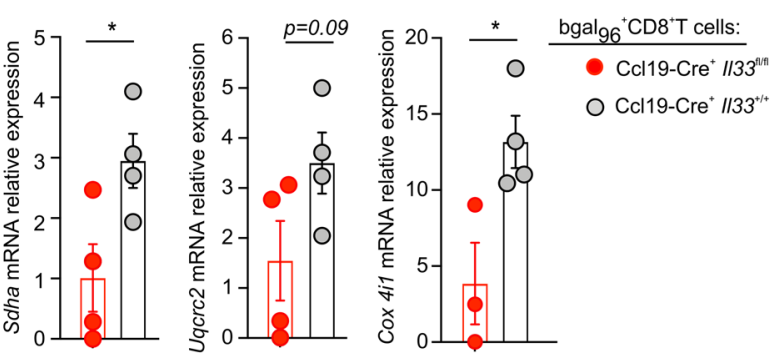

Extended Data Fig. 8 | Pulmonary FSC-derived IL-33 preserves metabolic and functional fitness of inflationary memory CD8 ${ }^{+} \mathbf{T}$ cells. (a to d, Ccl19-Cre-EYFP/iDTR mice were immunized i.v. with Ad-LacZ/FlexON and DT or PBS was given intranasally on day 3 and 5. Single cell RNA-seq analysis of bgal g $_{96}$ tetramer ${ }^{+} \mathrm{CD} 8{ }^{+} \mathrm{T}$ cells isolated from lungs on day 21. (a) Network plots displaying most significantly enriched gene ontologies based on transcriptional differences in bgal ${ }_{96}$ tetramer ${ }^{+} \mathrm{CD} 8+\mathrm{T}$ cells from DT vs PBS treated Ccl19-Cre/iDTR EYFP mice and with enriched genes annotated. (b) Violin plots show expression of Atp5b, Cox5a, Cox8a, Tomm20. (c) Representative FACS histograms of Ki67 staining on pulmonary bgal ${ }_{96}$ tetramer ${ }^{+}$ CD8 ${ }^{+} \mathrm{T}$ cells (left) and frequency of $\mathrm{Ki} 7^{+}$bgal $_{96}$-specific CD8 ${ }^{+} \mathrm{T}$ cells (right). (d) Representative FACS dot-plot of live-dead stain negative pulmonary bgal $_{96}$ tetramer ${ }^{+} \mathrm{CD} 8^{+} \mathrm{T}$ cells (left) and frequency of Aqua-negative bgal ${ }_{96}$-specific CD8 ${ }^{+} \mathrm{T}$ cells (right). e, Quantitative real-time PCR analysis of Sdha, Uqcrc2 and Cox4i2 expression in bgal ${ }_{96}$-specifc CD8 ${ }^{+} \mathrm{T}$ cells sorted from the lung of Ccl19-Cre $/ 133^{\mathrm{fl} / \mathrm{fl}}$ and Ccl19-Cre+ $/ 133^{+/+}$mice, day 21 after Ad-LacZ/ FlexON immunization. Dots represent individual mice. Bar graphs indicate mean \pm s.e.m. (c-e) Pooled data from 2 independent experiments with $n=8$ mice per group $[(\mathrm{c})] ; n=7$ mice $[(d)] ;$ and $n=4$ mice $[(e)]$. Statistical analysis was conducted using unpaired two-tailored Student's $t$ test with ${ }^{*} P<0.05$; ${ }^{\star \star} \mathrm{P}<0.01 ;{ }^{* \star \star} \mathrm{P}<0.001$; ${ }^{\star \star \star \star} \mathrm{P}<0.0001$. Exact $\mathrm{P}$ values are provided in the Source Data. 
a

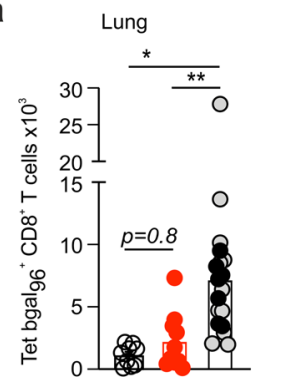

b

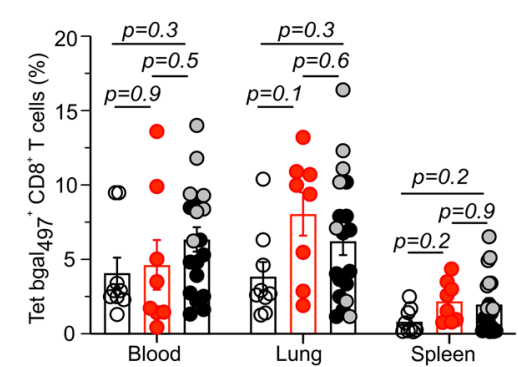

C

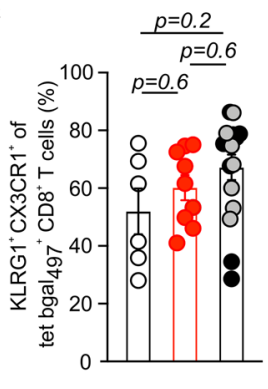

Ad-LacZ FlexON i.v. day 21

O CCl19-EYFP/iDTR DT i.n.

- Col19-EYFP/IDTR PBS i.n.

- Ccl19-Cre ${ }^{+} \| 33^{\text {nn }}$

O Col19-Cre+ $1133^{\text {th }}$

Extended Data Fig. 9 | Kinetics and tissue distribution of inflationary memory CD8 ${ }^{+} \mathrm{T}$ cells under conditions of Ccl19-Cre ${ }^{+}$lung FSC depletion and

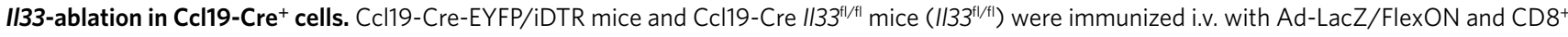
T cells were analyzed on day 21. Ccl19-Cre-EYFP/iDTR mice were treated i.n. with DT or PBS on day 3 and 5 after immunization. $\mathbf{a}$, Number of bgal ${ }_{96}$ tetramer ${ }^{+} \mathrm{CD} 8^{+} \mathrm{T}$ cells in lung and spleen. $\mathbf{b}$, Frequency of bgal ${ }_{497}$ tetramer ${ }^{+} \mathrm{CD} 8^{+} \mathrm{T}$ cells in blood, lung and spleen. $\mathbf{c}$, Frequency of KLRG1 ${ }^{+} / \mathrm{CX} 3 \mathrm{CR} 1^{+}$ bgal $_{497}$ tetramer ${ }^{+} \mathrm{CD} 8+\mathrm{T}$ cells in the lungs. d, IFN-g- and TNF-producing pulmonary bgal ${ }_{497}$-specific CD8 $^{+}$T cells. Dots represent individual mice. Bar graphs indicate mean \pm s.e.m. Pooled data from 2 independent experiments with $n=9$ (DT intranasal), $n=9\left(\right.$ Ccl19-Cre $\left./ / 33^{\mathrm{fl} / \mathrm{f}}\right)$ and $\mathrm{n}=19(\mathrm{Ccl} / 19-\mathrm{EYFP} /$ iDTR PBS i.n. and Ccl19-Cre $/ 133^{+/+}$) mice $[(a)] ; n=9$ (DT intranasal), $n=8$ (Ccl19-Cre $\left./ / 33^{f / / 1 / 1}\right)$ and $n=19$ (Ccl19-EYFP/iDTR PBS i.n. and Ccl19-Cre $/ / 33^{+/+}$) mice $[(b)] ; n=6$ (DT intranasal), $n=9$ (Ccl19-Cre $\left./ / 33^{f / / 1 / l}\right)$ and $n=16$ (Ccl19-EYFP/iDTR PBS i.n. and Ccl19-Cre $/ / 33^{+/+}$) mice $[(c)] ; n=7$ (DT intranasal), $\mathrm{n}=8 \mathrm{IFNg}, \mathrm{n}=6$ TNF (Ccl19-Cre $\left./ / 33^{\mathrm{f} / / 1 /}\right)$ and $\mathrm{n}=18$ (Ccl19-EYFP/iDTR PBS i.n. and Ccl19-Cre $/ / 33^{+/+}$) mice [(d)]. Statistical analysis was conducted using one-way ANOVA with Tukey's multiple comparison test with ${ }^{\star} P<0.05$; ${ }^{\star \star} P<0.01$; ${ }^{\star \star \star} P<0.001,{ }^{\star \star \star \star} P<0.0001$. Exact $P$ values are provided in the Source Data. 


\section{Reporting Summary}

Nature Research wishes to improve the reproducibility of the work that we publish. This form provides structure for consistency and transparency in reporting. For further information on Nature Research policies, see our Editorial Policies and the Editorial Policy Checklist.

\section{Statistics}

For all statistical analyses, confirm that the following items are present in the figure legend, table legend, main text, or Methods section.

n/a Confirmed

\ The exact sample size $(n)$ for each experimental group/condition, given as a discrete number and unit of measurement

\ A statement on whether measurements were taken from distinct samples or whether the same sample was measured repeatedly

The statistical test(s) used AND whether they are one- or two-sided

Only common tests should be described solely by name; describe more complex techniques in the Methods section.

\A description of all covariates tested

\A description of any assumptions or corrections, such as tests of normality and adjustment for multiple comparisons

$\checkmark$ A full description of the statistical parameters including central tendency (e.g. means) or other basic estimates (e.g. regression coefficient)

AND variation (e.g. standard deviation) or associated estimates of uncertainty (e.g. confidence intervals)

For null hypothesis testing, the test statistic (e.g. $F, t, r$ ) with confidence intervals, effect sizes, degrees of freedom and $P$ value noted Give $P$ values as exact values whenever suitable.

Х $\square$ For Bayesian analysis, information on the choice of priors and Markov chain Monte Carlo settings

$\bigotimes \square$ For hierarchical and complex designs, identification of the appropriate level for tests and full reporting of outcomes

$\square \bigotimes$ Estimates of effect sizes (e.g. Cohen's $d$, Pearson's $r$ ), indicating how they were calculated

\section{Our web collection on statistics for biologists contains articles on many of the points above.}

\section{Software and code}

Policy information about availability of computer code

Data collection FACSDiva (BD Bioscience, v8.0.1), FACSChorus (BD Biosciences, v1.3), ZEN (Zeiss, v14.0.18.201), The QuantStudioTM Design \& Analysis Software v1.5.1

Data analysis FlowJo (Treestar Inc., v10), Prism (Graphpad, v8.4.3), R Studio ( v 3.6.1), Cell Ranger (v 3.0.2), R packages running in R v3.6.1: scater (v1.14.1), Seurat (v3.1.1), clusterProfiler (v.3.16.0), SeuratWrappers (v.0.1.0), velocyto.py (v.0.17.17), Imaris (v7 and v8)

For manuscripts utilizing custom algorithms or software that are central to the research but not yet described in published literature, software must be made available to editors and

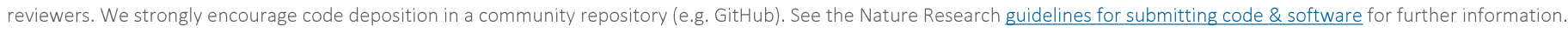

\section{Data}

Policy information about availability of data

All manuscripts must include a data availability statement. This statement should provide the following information, where applicable:

- Accession codes, unique identifiers, or web links for publicly available datasets

- A list of figures that have associated raw data

- A description of any restrictions on data availability

ScRNA-seq data are available in ArrayExpress database (accession numbers E-MTAB-9558 and E-MTAB-9580). Ensembl GRCm38.94 was used as reference to build index files for alignments in scRNA-seq analysis. Further information and requests for resources should be directed to and will be fulfilled by the lead contacts. 
Please select the one below that is the best fit for your research. If you are not sure, read the appropriate sections before making your selection.

$\bigotimes$ Life sciences $\quad \square$ Behavioural \& social sciences $\quad \square$ Ecological, evolutionary \& environmental sciences

For a reference copy of the document with all sections, see nature.com/documents/nr-reporting-summary-flat.pdf

\section{Life sciences study design}

All studies must disclose on these points even when the disclosure is negative.

$\begin{array}{ll}\text { Sample size } & \begin{array}{l}\text { No sample-size calculation was performed; sample size were determined to be adequate based on the reproducibility between independent } \\ \text { experiments. }\end{array} \\ \text { Data exclusions } & \text { No data point were excluded. } \\ \text { Replication } & \begin{array}{l}\text { Experiments were repeated at least two times. Animal experiments were repeated with sufficient animals per group to demonstrate statistical } \\ \text { significance. All experiments were reliably reproduced. }\end{array} \\ \text { Randomization } & \begin{array}{l}\text { Subjects were randomly assigned to experimental groups. For animal experiments, six to ten weeks old female and male mice were age } \\ \text { matched and randomly assigned to experimental groups. }\end{array} \\ \text { Blinding } & \begin{array}{l}\text { Blinding was not performed in this study. Data analysis was strictly quantitative, with objective outcomes therefore blinding was not relevant } \\ \text { for this study. }\end{array}\end{array}$

\section{Reporting for specific materials, systems and methods}

We require information from authors about some types of materials, experimental systems and methods used in many studies. Here, indicate whether each material, system or method listed is relevant to your study. If you are not sure if a list item applies to your research, read the appropriate section before selecting a response.

\begin{tabular}{|c|c|c|c|}
\hline \multicolumn{2}{|r|}{ Materials \& experimental systems } & \multicolumn{2}{|c|}{ Methods } \\
\hline $\mathrm{n} / \mathrm{a}$ & Involved in the study & $\mathrm{n} / \mathrm{a}$ & Involved in the study \\
\hline$\square$ & $\bigotimes$ Antibodies & $\bigotimes$ & $\square$ ChIP-seq \\
\hline $\mathbf{L}^{-1}$ & $\bigotimes$ Eukaryotic cell lines & $\square$ & $\bigotimes$ Flow cytometry \\
\hline$\bigotimes$ & $\square$ Palaeontology and archaeology & Х & $\square$ MRI-based neuroimaging \\
\hline 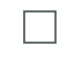 & $\bigotimes$ Animals and other organisms & & \\
\hline L & $\bigotimes$ Human research participants & & \\
\hline$\bigotimes$ & $\square$ Clinical data & & \\
\hline Х & $\square$ Dual use research of concern & & \\
\hline
\end{tabular}

\section{Antibodies}

Antibodies used

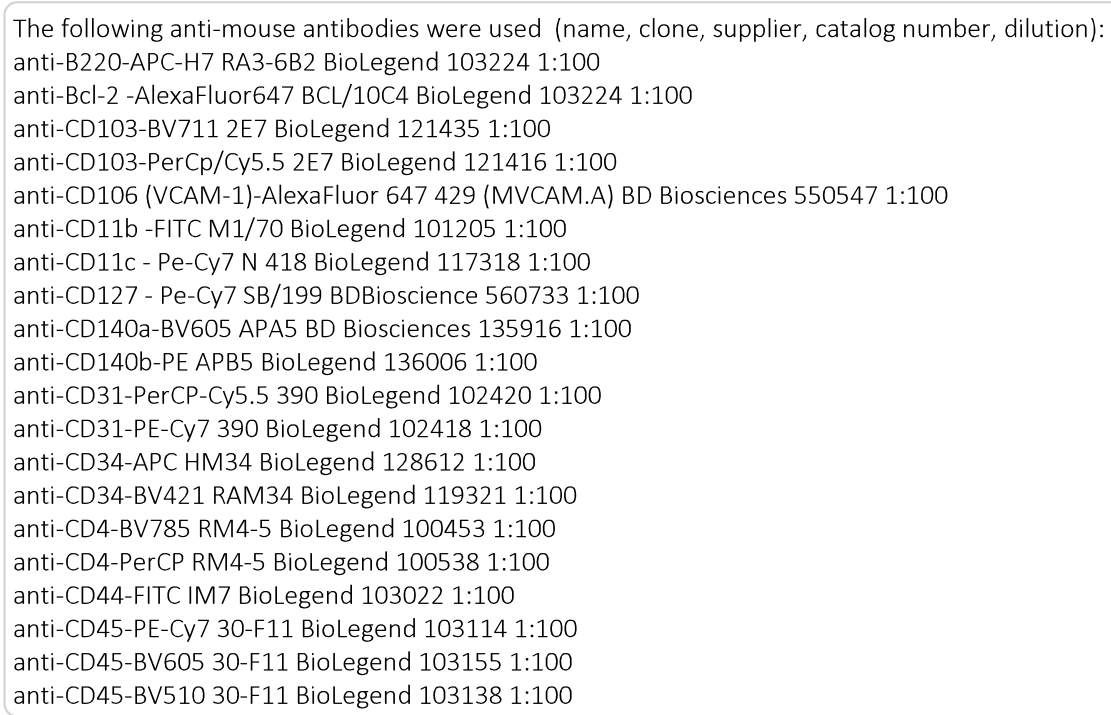




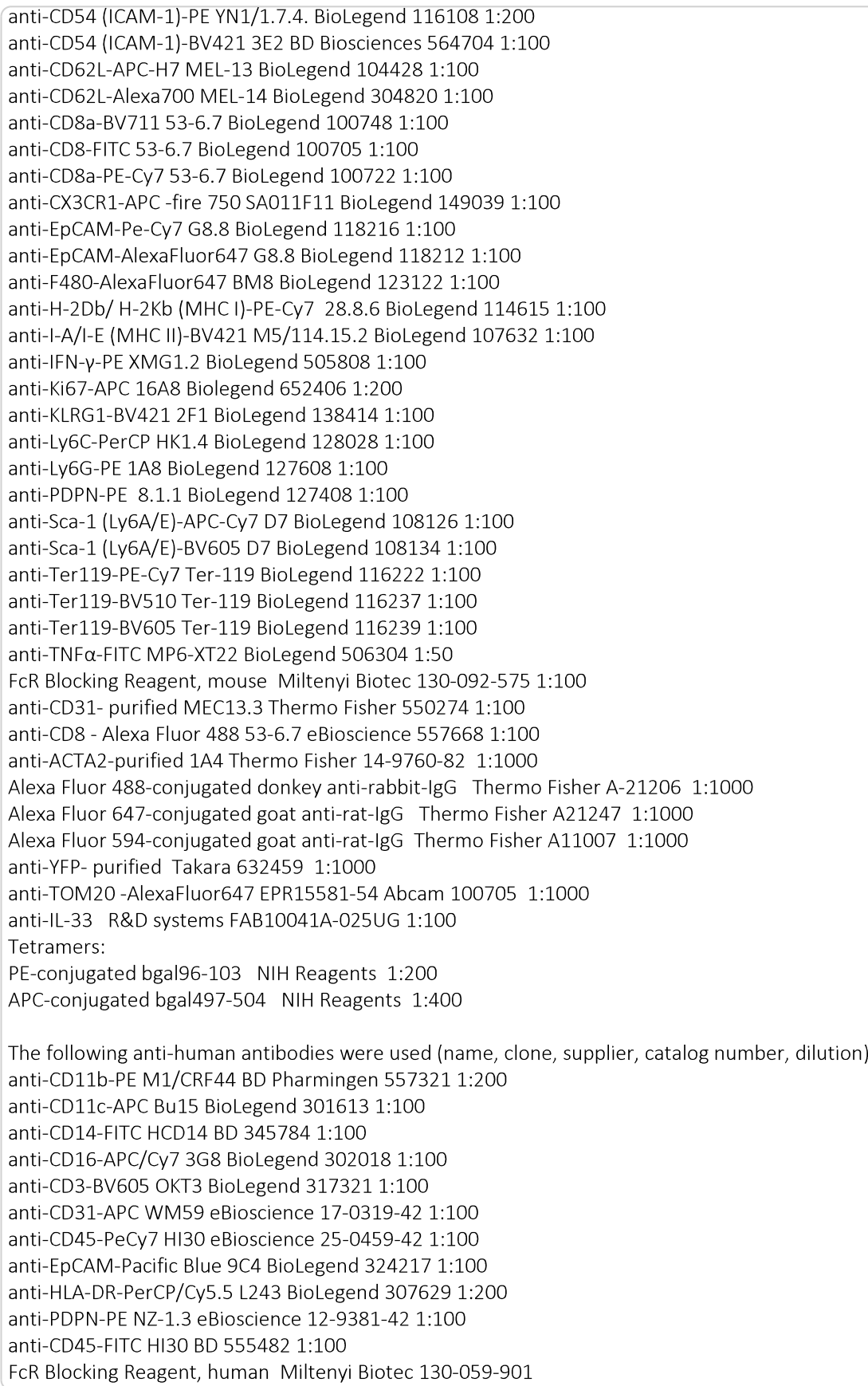

Validation

All mouse and human antibodies except tetramer PE-conjugated bgal96-103 and APC-conjugated bgal497-504 came from commercial vendors and have been validated by the manufacturers on their official websites.

\section{Eukaryotic cell lines}

Policy information about cell lines

Cell line source(s)

\section{Authentication}

Mycoplasma contamination

Commonly misidentified lines (See $\underline{I C L A C}$ register)
The murine B16 melanoma LacZ-expressing cell line (B16-LacZ) was obtained from RIKEN BRC (Japan).

Cell line was not authenticated in out laboratory.

B16-LacZ tested negative for mycoplasma contamination in our laboratory.

No misidentified cell lines were used.

\section{Animals and other organisms}

Policy information about studies involving animals; ARRIVE guidelines recommended for reporting animal research 

and LysM-Cre mice were purchased from Jackson Laboratory. H2-Kb -/- mice were purchased from Taconic Farms. R26R-iDTR (C57BL/6-Gt[ROSA]26Sortm1 [HBEGF]Awai/J) were obtained from A. Waisman (University of Mainz, Germany), and Cd11C-Cre mice were obtained from B. Reizis (New York University School of Medicine, USA) and Ubi-Cre ERT2 mice were obtained from T Vincent (Kennedy Institute, University of Oxford). The II33 targeting vector to generate the II33fl/fl mice was generated by the trans-NIH Knock-Out Mouse Project (KOMP Project ID CSD88909) and obtained from the KOMP Repository (www.komp.org). To ablate II33 expression in a distinct FSC subset, Ccl19-Cre mice were crossed with II33fl/fl mice. Ccl19-Cre mice were bred to R26R-EYFP or Rosa26R-iDTR and R26R-EYFP mice to generate heterozygous Ccl19-Cre R26R-EYFP and Ccl19-Cre R26R -EYFP/iDTR mice. Batf3-/mice were obtained from M Kopf (ETH Zurich, Switzerland). All mice were housed in the Institute of Immunobiology, Kantonsspital St. Gallen under specific-pathogen-free (SPF) conditions at $22^{\circ} \mathrm{C}$ and $30-70 \%$ humidity in a $12 / 12-\mathrm{h}$ light/dark cycle and provided ad libitum access to food and water. All experiments have been performed with 7 to 10 weeks old mice (males and females).

Wild animals

None

Field-collected samples

None

Ethics oversight

Experiments were performed in accordance with federal and cantonal guidelines (Tierschutzgesetz) of Switzerland under permission numbers SG08/17, SG01/18, SG07/19, SG04/20 and SG01/20 following review and approval by the respective Cantonal Veterinary Offices (St. Gallen, Switzerland).

Note that full information on the approval of the study protocol must also be provided in the manuscript.

\section{Human research participants}

Policy information about studies involving human research participants

Population characteristics

Tonsillar material was collected from healthy male and female children age between 3 and 14 years that are undergoing routine tonsillectomy. Informed consent signed by at least one parent or the legal guardian for children from $3-13$ years of age, for 14-year-old children, the child also signed the informed consent. Subjects presenting the following features have been excluded from the study: 1) Major craniofacial abnormalities, 2) Undergoing immunosuppression or antibiotic treatment, 3) Major inflammatory lesions on the tonsils, 4) Patients with the pre-existing immunocompromising disease. Healthy volunteers (age 25-35) donated blood. Lung tissue obtained from a non-small cell lung cancer patient (male) undergoing debulking surgery of lung cancer and adjacent unaffected lung tissue has been obtained for the analysis. Skin tissue was obtained by a healthy patient undergoing reduction mammaplasty (female). All adult patients have signed informed consent.

Recruitment Patient samples were obtained from patients of the Children's Hospital St. Gallen, the Institute of Pathology at the Kantonsspital St Gallen and healthy volunteers. Biological material collected from healthy children undergoing routine tonsillectomy in the Children 's Hospital St. Gallen. Lung samples were obtained from the Institute of Pathology at the Kantonsspital St Gallen after obtaining informed consent from the patients. The patients were undergoing debulking surgery of lung cancer and adjacent unaffected lung tissue has been obtained for the analysis. Skin tissue was obtained by a healthy patient undergoing reduction mammaplasty. There was no self-selection bias or investigator selection bias in determining which subjects were included in the study or in the data analysis.

Ethics oversight

The study has been approved by the ethics committee of Eastern Switzerland as EKOS 18/113 (Assessment of target cell tropism of viral vectors in tonsillar explants), the St. Gallen Lung Biopsy Biobank (EKSG 11/044) and within the frame of the general consent at the Kantonsspital St.Gallen

Note that full information on the approval of the study protocol must also be provided in the manuscript.

\section{Flow Cytometry}

Plots

Confirm that:

\The axis labels state the marker and fluorochrome used (e.g. CD4-FITC).

\The axis scales are clearly visible. Include numbers along axes only for bottom left plot of group (a 'group' is an analysis of identical markers).

$\bigotimes$ All plots are contour plots with outliers or pseudocolor plots.

$\bigotimes$ A numerical value for number of cells or percentage (with statistics) is provided.

\section{Methodology}

Sample preparation

Instrument

Software

Cell population abundance
A description of sample preparation for the flow cytometry is detailed in the methods section.

LSR Fortessa 2 BD Bioscience, FACS Melody BD Bioscience

FACS Diva was used to collect the data and FlowJo (Treestar Inc.) to analyze the data. For cell sorting for scRNA seq, FACSChorus was used to set up cell sorting. R studio was used to analyze transcriptomic data.

Cells were checked for purity of sorted populations after flow cytometry assisted cell sorting using LSR Fortessa 2. 
For all flow cytometric analysis, cells were first gated on FSC/SSC to exclude cell debris following by FSC-A/FSC-H to exclude doublets. Dead cells were excluded from analysis by gating on viability dye negative staining. Gating strategy for identifying pulmonary fibroblastic stromal cells and tetramer+ CD8+ T cells analyzed in this study is exemplifying in the Extended Data Figures.

$\bigotimes$ Tick this box to confirm that a figure exemplifying the gating strategy is provided in the Supplementary Information. 